Arqueología y Sociedad,

№ 20, 2009

\title{
SiSTEMA DE VALORACIÓN DE MONUMENTOS ARQUEOLÓGICOS EN PLANES DE MANEJO. EL CASO DEL PLAN MAESTRO DE MANEJO Y CONSERVACIÓN DE KUELAP Y SU ENTORNO
}

\begin{abstract}
Aldo Bolaños*
Resumen

Se presenta el sistema de valoración de monumentos arqueológicos desarrollado para el Plan maestro de manejo y conservación de Kuelap y su entorno (PMK) en el año 2003. La importancia del artículo reside en la posibilidad de incluir nuevos parámetros descriptivos de patrimonio arqueológico en función a la necesidad de desarrollar planes de gestión que garanticen su conservación y que regulen las intervenciones que en ellos se realicen. Se muestran los aspectos metodológicos del sistema de valoración utilizado y sus principales resultados en figuras y gráficos detallados para la discusión acerca de la validez del método, tomando en cuenta que no existen referencias previas para el sistema utilizado en el Perú. El artículo presenta los sitios identificados, el ordenamiento de la información y la calificación de los sitios en base a sus limitaciones, potenciales y las actividades requeridas, el establecimiento de las Unidades Geohistóricas (UGH), y al desarrollo de plan de intervenciones priorizadas por cada sitio y UGH.
\end{abstract}

\section{Palabras claves}

Sistema de valoración de monumentos arqueológicos, gestión cultural, planes de manejo, Kuelap, Utcubamba, áreas agroarqueológicas.

\begin{abstract}
There appears the system of evaluation of archaeological monuments developed for the Main Plan of Handling and Conservation of Kuelap and his environment (PMK) in 2003. The importance of the article resides in the possibility of including new descriptive parameters of archaeological patrimony in function to the need to develop plans of management that guarantee his conservation and that regulate the interventions that in them are realized. There appear the methodological aspects of the used system of evaluation and his principal results in figures and graphs detailed for the discussion about the validity of the method, taking into consideration that there do not exist previous references to the system used in Peru. The article presents the identified places, the arranging of the information and the qualification of the places based on his limitations, potentials and the required activities, the establishment of the Geohistorical Units $(\mathrm{UGH})$, and the development of plan of interventions prioritized by every place and $\mathrm{UGH}$.
\end{abstract}

\section{Keywords}

System of evaluation of archaeological monuments, cultural management, plans of handling, Kuelap, Utcubamba, agroarqueological areas.

\footnotetext{
* Arqueólogo. Licenciado en la Escuela de Arqueología de la Facultad de Ciencias Sociales de la Universidad Nacional Mayor de San Marcos. Miembro asociado del Instituto Andino de Estudios Arqueológicos - INDEA (1984 - 1993); Miembro fundador y Director y de la Asociación Arqueología para el Desarrollo (1994 - 1997); Miembro fundador del Equipo Peruano de Antropología Forense - EPAF desde 1997 y Miembro del Comité Directivo (2003 - 2008); Miembro Fundador de la Asociación Latinoamericana de Antropología Forense - ALAF desde el 2003 y Miembro del Comité Directivo 2005 - 2008. Actualmente el autor lleva adelante la Iniciativa Paisaje y Memoria. Correo electrónico: : aldofernando@yahoo.com
} 
El año 2003, como miembro del equipo de elaboración del Plan Maestro Kuelap $(\mathrm{PMK})^{1}$ propuse un sistema de valoración de monumentos arqueológicos que permitiera abordar las tareas relativas a la planificación de las investigaciones, la conservación y el equipamiento de los sitios y áreas arqueológicas comprendidas en el territorio de estudios. El sistema de valoración es un requisito indispensable para la realización de planes de manejo del patrimonio cultural en el largo plazo como el PMK (diez años).

Sin embargo, aparte del reto del diseño del sistema (hasta ese momento inexistente) hubo también que enfrentar las limitaciones que las tradicionales descripciones arqueológicas, tanto de investigadores particulares como las propuestas por el propio Instituto Nacional de Cultura (INC), presentaban para la proyección de actividades. Se sumaba a esto, la inexistencia de medios automatizados de sistematización y manejo de información especializada adaptada a las necesidades del plan.

El sistema de valoración comprendió cuatro fases de trabajo: en la primera, se realizó la identificación de los sitios arqueológicos con dos tareas básicas: la documentación de las fuentes bibliográficas y cartográficas del área de trabajo -la que continuó a lo largo de toda la investigación- y la exploración y registro de campo de los sitios y áreas arqueológicas ${ }^{2}$. La segunda fase consistió en el ordenamiento de la información y la calificación de los sitios arqueológicos a partir de un sistema de cuantificación con valores numéricos del estado de conservación y los factores de riesgo existentes de los sitios y áreas arqueológicas y al mismo tiempo, la cuantificación de los factores potenciales para la puesta en uso social de los monumentos. Para esta tarea, se examinaron también los procesos económicos, sociales, antropológicos, geográficos así como el papel de las instituciones sectoriales que influyen en los factores de riesgo y potencial del patrimonio arqueológico regional. La tercera fase consistió en el establecimiento de las Unidades Geohistóricas (UGH), las cuales surgieron como producto de la discusión del equipo y a partir de las cuales se agruparon los sitios para la realización de su selección, priorización y determinación del tipo de intervención. La cuarta y última fase se dedicó, en base a todo el proceso anterior, al desarrollo de plan de intervenciones priorizadas por cada sitio y UGH. Finalmente, la quinta fase se dedicó a la elaboración de 3 perfiles de proyectos y un plan general de intervención para el área del PMK.

Como es posible comprender, los informes realizados abarcaron un número de páginas importante que trasciende las posibilidades de esta publicación, sin embargo, se pretende acá presentar un resumen de los principales resultados en los aspectos metodológicos de la evaluación, calificación, selección, priorización utilizados proponiendo un primer modelo de sistematización de la información para este tipo de investigaciones.

\section{IDENTIFICACIÓN DE LOS SITIOS ARQUEOLÓGICOS}

La identificación de los sitios fue realizada a través de: a) la revisión de las fuentes bibliográficas y cartográficas; b) la referencia verbal de personas de la zona con interés en la promoción de su patrimonio cultural local (María, Magdalena, Montevideo Jalca Grande) a partir de entrevis-

1 El Plan maestro de manejo y conservación de Kuelap y su entorno (PMK) fue elaborado entre los años 2003 y 2004 como una iniciativa entre el Ministerio de Comercio Exterior y Turismo - MINCETUR, PROINVERSION y el Instituto Nacional de Cultura - INC. El trabajo estuvo dirigido por el arqueólogo Alfredo Narváez. El área del PMK abarcaba un total de diez distritos entre el poblado de Tingo y el de Leymebamba ( $35 \mathrm{~km}$ de largo por un ancho promedio de $5 \mathrm{~km}$ hacia cada margen de la cuenca), ubicados en el alto Utcubamba.

2 Para la exploración se diseñó una ficha adaptada a las necesidades del plan. Dicha ficha sería mejorada los años 2005 - 2006 y propuesta a los equipos de planificación arqueológica del Programa Qhapaq Ñan del INC y a los encargados de la elaboración del Plan de Acción Regional del mismo programa; la ficha y su guía de uso será presentada en un futuro artículo. 


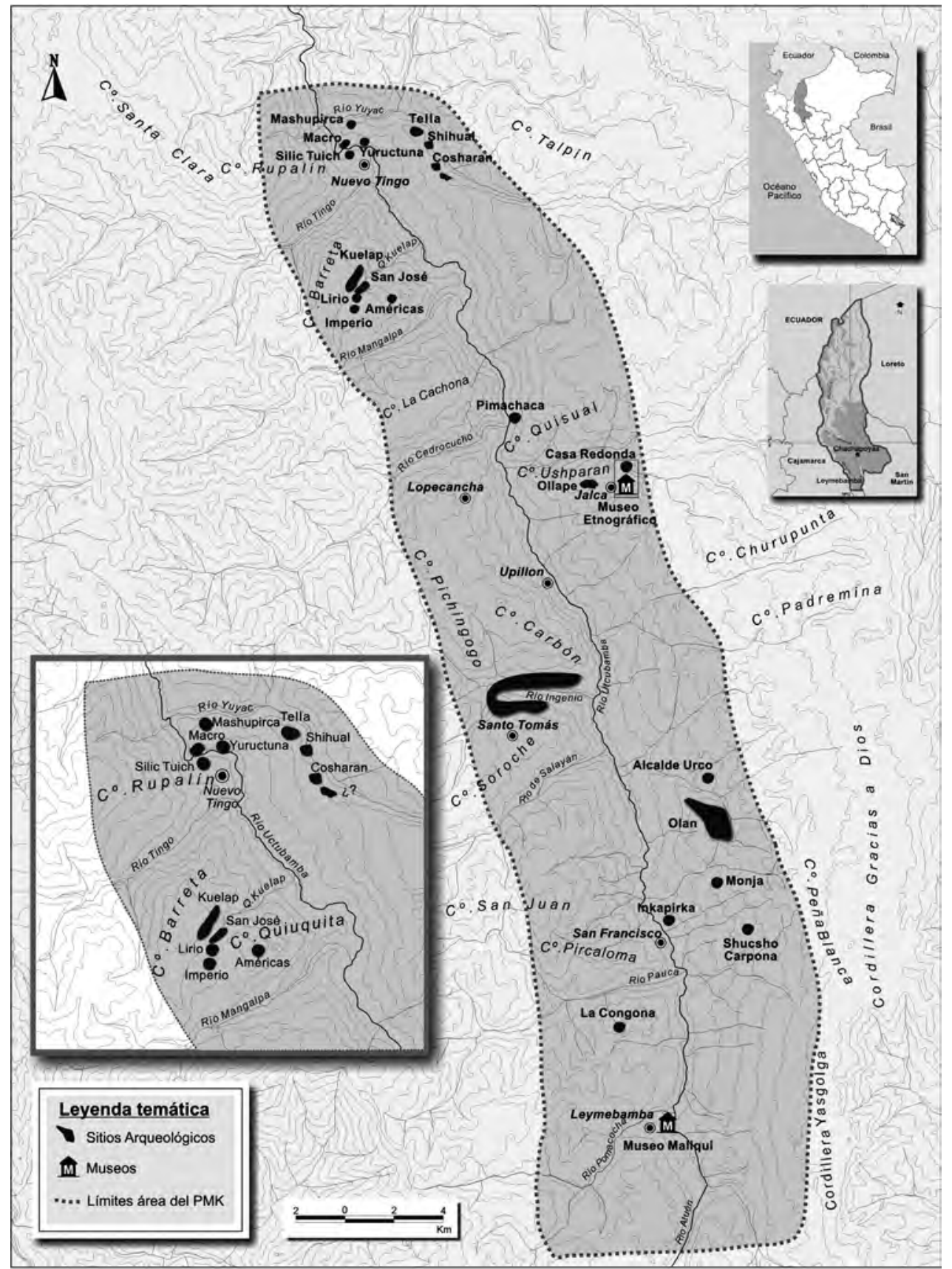

Mapa arqueológico de la zona del Plan Maestro Kuelap elaborado el 2003 con 23 de los sitios arqueológicos ubicados. 
tas personales y las referencias obtenidas en los talleres locales; c) la visita de identificación (del 10 al 21 de febrero y del 6 de junio al 14 de junio 2003)

En el área comprendida por el PMK se ubicaron 35 sitios, de ellos 32 corresponden a asentamientos (aldeas fortificadas, aldeas y estructuras funerarias) 2 son áreas agroarqueológicas y 3 secciones de caminos prehispánicos (Cuadro 1). 24 de los sitios fueron visitados por el PMK; 14 estaban incluidos en el mapa Diagnóstico Turístico del Microcorredor Socio Económico Kuelap del Sistema de Información Geográfica del CTAR (Diciembre 2002; 1:150,000) De los 35 sitios y áreas arqueológicas, 16 ya habían sido declarados Patrimonio Cultural de la Nación (Resolución Directoral Nacional N 196/INC del 02 de abril del 2003) y quedaban aun por realizar los expedientes técnicos para su correcta ubicación y descripción de 19 de los sitios (Tabla 1).

En 9 de los 10 distritos que conforman el PMK se identificaron en las fuentes y referencias orales, un total 158 sitios arqueológicos. Sin embargo, ya que la exploración de campo contó con un tiempo muy reducido (20 días), no se pudo determinar cuántos de los sitios identificados se encontraban dentro del área del plan Maestro. Como parte del PMK se propuso su verificación y evaluación posterior como parte de un Proyecto de Inventario y Catastro de Sitios Arqueológicos en visitas de campo más largas y mayores investigaciones bibliográficas, a fin de evitar el impacto que el PMK podría tener en ellos al aumentar la actividad turística regional.

El distrito que contó con mayor número de sitios arqueológicos registrados fue el de La Jalca. Al momento, este mayor número de sitios no puede ser tomado como indicativo de un mayor poblamiento prehispánico, sino más bien como producto de un mejor conocimiento del lugar por parte de algunos personajes locales (Tulio Cullqui).

La identificación de los sitios arqueológicos (fig. 1) presentó un conjunto de problemas que se detallan a continuación: los sitios se encontraban mencionados en fuentes dispersas y ninguna de ellas en forma completa. En el caso de las fuentes bibliográficas sólo 12 de los sitios fueron identificados a partir de referencias en informes y publicaciones de investigación arqueológica (Kuelap, Revash, Macro, Shihual, Plazapampa, Gupji, Cueva del Chivo, Olán, y Alcalde Urco, Secsto, Putquero Loma, Arica). Aparte de Kuelap, el INC de Amazonas sólo contaba con informes institucionales de 7 sitios arqueológicos; 5 como parte del complejo arqueológico Kuelap (La Petaca, La barreta, San José, Lirio, Imperio), 1 del sitio de Carpona y 1 del sitio de Tuich/Silic. Al momento de la realización del proyecto, el INC de Amazonas no contaba con un inventario oficial de sitios arqueológicos para el área de estudio y en general, para todo el Departamento. Los planes de desarrollo turístico de Amazonas incluían fichas descriptivas para 13 de los sitios arqueológicos más conocidos y visitados; las fichas se repiten en varios de esos informes y no pasaban de ser mínimas e insuficientes para los objetivos de un Plan Maestro.

La mayoría de los 158 sitios arqueológicos identificados por el PMK correspondieron a las referencias dadas por personas de la localidad, especialmente en los casos de Montevideo y Jalca grande. El número de sitios de referencia oral fue de 65 y por medio de los talleres locales 34. Se trataba de la mayoría de los sitios identificados.

La cartografía consultada era igualmente incompleta, en muchos casos sin coordenadas geográficas y con ubicaciones referenciales. El Mapa Arqueológico del Departamento de Amazonas, elaborado por el Instituto Nacional de Cultura (INC 2000), solamente mencionaba sitios y no era un mapa útil para las tareas de ubicación, acceso, descripción y registro del patrimonio arqueológico de la región. Aparte de estos mapas se consultó también el Mapa Diagnóstico Turístico del Microcorredor Socio Económico Kuelap del Sistema de Información Geográfica del CTAR (Diciembre 2002; 1:150,000). Sólo 
Figura 1: Identificación de sitios arqueológicos en el área del PMK

\begin{tabular}{|c|c|c|c|c|}
\hline Sitio & Distrito & Visitado & Fuentes & Declaración INC \\
\hline 1. Kuelap & Tingo & $\mathrm{X}$ & CTAR 2002 & $\mathrm{X}$ \\
\hline 2. Lirio & Tingo & $\mathrm{X}$ & Informes INC & $\mathrm{X}$ \\
\hline 3. San José & Tingo & $\mathrm{X}$ & Informes INC & - \\
\hline 4. Imperio & Tingo & $\mathrm{X}$ & Informes INC & - \\
\hline 5. Las Américas & Tingo & - & Informes INC & - \\
\hline 6. Tuich//Silic & Tingo & $\mathrm{X}$ & Informes INC & $\mathrm{X}$ \\
\hline 7. Santa Clara & Tingo & - & - & - \\
\hline 8. Macro & Magdalena & $\mathrm{X}$ & CTAR 2002 & $\mathrm{X}$ \\
\hline 9. Machupirca & Magdalena & $\mathrm{X}$ & CTAR 2002 & - \\
\hline 10. Tella & Magdalena & $\mathrm{X}$ & CTAR 2002 & - \\
\hline 11. Qhapaq Nan & Magdalena & - & RTDA & - \\
\hline 12. Yuructuna & Magdalena & $\mathrm{X}$ & Jesús Culqui & - \\
\hline 13. Pumachaca & Magdalena & $\mathrm{X}$ & Jesús Culqui & - \\
\hline 14. Shihual & Magdalena & $\mathrm{X}$ & CTAR 2002 & - \\
\hline 15. Olán & M. Castilla & $\mathrm{X}$ & CTAR 2002 & $\mathrm{X}$ \\
\hline 16. Alcalde Urco & M. Castilla & - & CTAR 2002 & $\mathrm{X}$ \\
\hline 17. Revash & Santo Tomás & $\mathrm{X}$ & CTAR 2002 & $\mathrm{X}$ \\
\hline 18. Arica/Carpona & Montevideo & - & CTAR 2002 & $\mathrm{X}$ \\
\hline 19. Incapirka & Montevideo & $\mathrm{X}$ & CTAR 2002 & $\mathrm{X}$ \\
\hline 20. Monja & Montevideo & - & CTAR 2002 & $\mathrm{X}$ \\
\hline 21. Palpata & Montevideo & - & CTAR 2002 & $\mathrm{X}$ \\
\hline 22. Shucsho & Montevideo & $\mathrm{X}$ & - & - \\
\hline 23. Carpona & Montevideo & - & Liborio Tafur; MAA & $\mathrm{X}$ \\
\hline 24. Ollape & Jalca Grande & $\mathrm{X}$ & CTAR 2002 & $\mathrm{X}$ \\
\hline 25. Casa Redonda & Jalca Grande & $\mathrm{X}$ & CTAR 2002 & $\mathrm{X}$ \\
\hline 26. Putquero Loma & Jalca Grande & - & MAA & - \\
\hline 27. Sec'sto & Jalca Grande & - & MAA & $\mathrm{X}$ \\
\hline 28. Qapac Nan & Jalca Grande & $\mathrm{X}$ & Tulio Culqui & - \\
\hline 29. Camino La Jalca Ubillón & Jalca Grande & $\mathrm{X}$ & Tulio Culqui & - \\
\hline 30. La Congona & S. F. del Yeso & $\mathrm{X}$ & CTAR 2002 & $\mathrm{X}$ \\
\hline 31. Cataneo & S. F. del Yeso & $\mathrm{X}$ & ICRM.PMK & - \\
\hline 32. El Molinete & S. F. del Yeso & $\mathrm{X}$ & ICRM.PMK & - \\
\hline 33. Challuapunta & S. F. del Yeso & - & - & - \\
\hline 34. Andenes & La Jalca & $\mathrm{X}$ & - & - \\
\hline 35. Andenes & M. Castilla & $\mathrm{X}$ & - & - \\
\hline SUBTOTAL & & 24 & & 16 \\
\hline
\end{tabular}


dos sitios, Kuelap y Revash, se encontraban, ubicados oficialmente en la Carta Nacional 1:100,000. Se encontró también que los nombres de los sitios en los mapas no correspondían con las denominaciones que los pobladores del lugar le daban a éstos.

A estas limitaciones en la documentación se sumaron los problemas de campo. Aparte del corto tiempo de trabajo de campo, los árboles, arbustos y malezas cubrían los sitios arqueológicos demorando o impidiendo la realización del reconocimiento y las descripciones arqueológicas completas de aquellos sitios visitados por el PMK. Dimensionar su extensión y área con precisión y por lo tanto, definir las áreas de uso social y protección de los sitios, requiere aún superar este factor limitante. Se concluyó que los reconocimientos arqueológicos debían ser ampliados, sin embargo, saltaba a la vista rápidamente el potencial arqueológico, educativo y cultural del patrimonio cultural en el área del PMK.

\section{EVALUACIÓN DEL ESTADO DE CONSERVACIÓN DE} LOS SITIOS Y LAS COLECCIONES ARQUEOLÓGICAS Sobre la base de las descripciones de los sitios arqueológicos se estableció la prioridad de los problemas detectados para su solución y manejo por el Plan Maestro Kuélap (PMK). Estos problemas fueron organizados en ámbitos distintos: problemas de primer, de segundo y de tercer orden. Los problemas de primer orden se refieren a aquellos que afectan en forma directa y negativa la conservación de los restos arqueológicos y son descritos en las figuras 2 y 3 . Los problemas de segundo orden se refieren a aquellos factores que propician o permiten que las situaciones negativas ocurran causando su deterioro y son descritos en las figuras 4 y 5 . El tercer orden de problemas abarca todos aquellos producidos como resultado de procesos sociales de causas y soluciones complejas, pero que influyen decididamente sobre los sitios arqueológicos. Estos tres grupos de problemas interactúan formando cadenas de situaciones que requieren del manejo integral de las soluciones ${ }^{3}$.

\subsection{Problemas de Primer Orden}

Figura 2. Causas de la destrucción de sitios y áreas arqueológicas. Problemas de primer orden

\begin{tabular}{|c|c|c|c|c|c|c|c|c|c|c|c|}
\hline \multirow[t]{2}{*}{ Sitios } & \multicolumn{3}{|c|}{ Naturales } & \multicolumn{6}{|c|}{ Antrópicas } & & \multirow[t]{2}{*}{ Total } \\
\hline & $\frac{\frac{\pi}{N}}{\sum^{\pi}}$ & 总 & 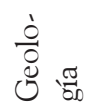 & 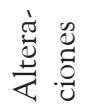 & 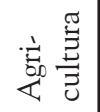 & 总 & $\stackrel{\dot{\jmath}}{a} \stackrel{\approx}{g}$ & $\stackrel{\circ}{\mathscr{m}}$ & $\begin{array}{l}\frac{\pi}{\pi} \\
\stackrel{\tilde{\sigma}}{\sigma} \stackrel{\infty}{0}\end{array}$ & $\frac{\mathscr{\pi}}{0}$ & \\
\hline Kuelap & $\mathrm{X}$ & $\mathrm{X}$ & $X$ & $\mathrm{X}$ & & & $\mathrm{X}$ & & & & 5 \\
\hline Lirio & & $\mathrm{X}$ & & $\mathrm{X}$ & $X$ & & & & $X$ & & 4 \\
\hline San José & $X$ & $\mathrm{X}$ & & & $X$ & & & & & & 3 \\
\hline Imperio & $X$ & $X$ & & $\mathrm{X}$ & $X$ & $\mathrm{X}$ & & & & & 5 \\
\hline Las Américas & - & - & - & - & - & - & - & - & - & - & - \\
\hline Tuich / Silic & $X$ & $\mathrm{X}$ & $X$ & $\mathrm{X}$ & $\mathrm{X}$ & & & & & $\mathrm{X}$ & 6 \\
\hline Santa Clara & - & - & - & - & - & - & - & - & - & - & - \\
\hline Macro & $X$ & $\mathrm{X}$ & $\mathrm{X}$ & & & & & & & & 3 \\
\hline Machupirca & $\mathrm{X}$ & $\mathrm{X}$ & $X$ & $\mathrm{X}$ & $\mathrm{X}$ & & $\mathrm{X}$ & & & & 6 \\
\hline
\end{tabular}

3 Se presenta un resumen de las problemáticas encontradas a lo largo del proyecto a fin de contextuar el sistema de valoración que se describe más adelante. Se reserva la publicación completa del análisis de problemáticas para un nuevo artículo. 


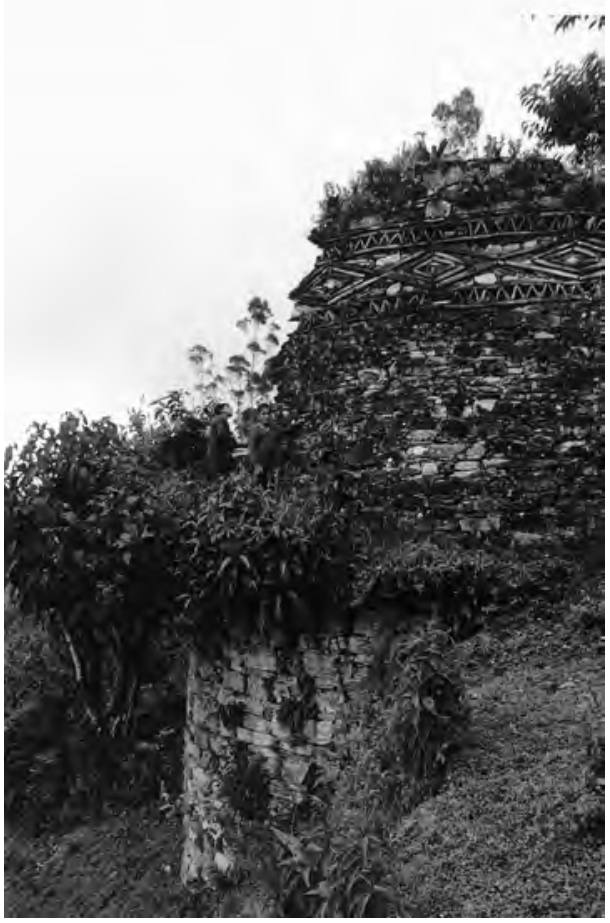

Fotografía 1. Olán, distrito de Montevideo, estructura circular de $10 \mathrm{~m}$ de altura con decoración exterior.

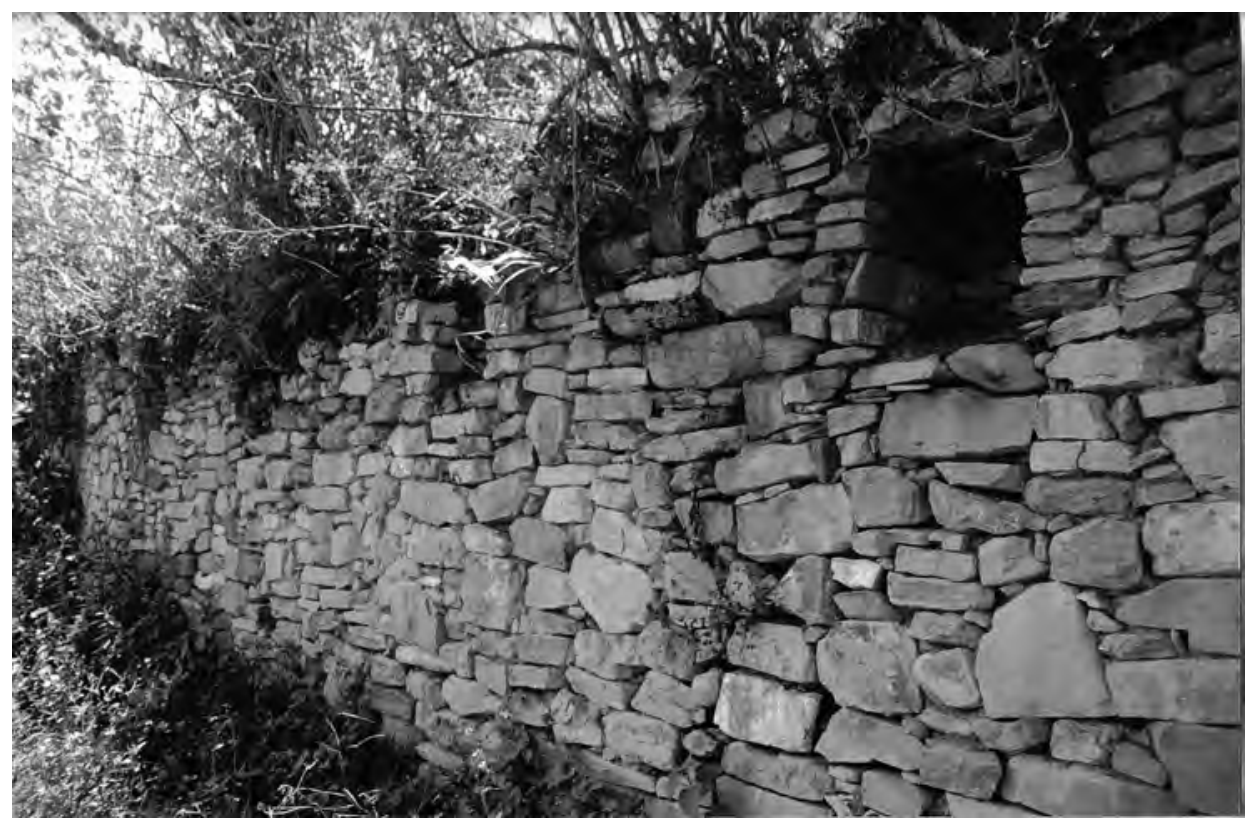

Fotografía 2. Qhapaq Ñan en Ubilón. Paredes laterales de viaducto con hornacinas en la parte superior. 


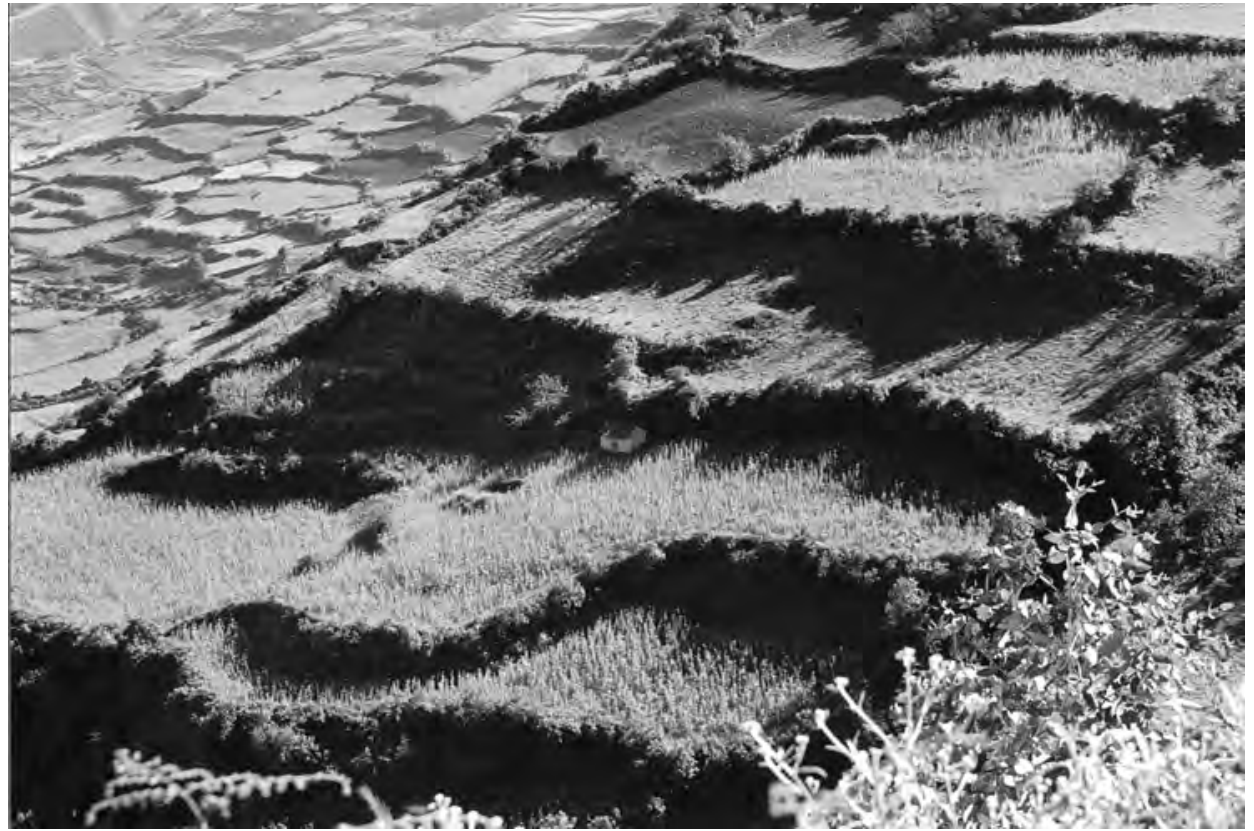

Fotografía 3. Zona agroarqueológica en Montevideo.

Andenes cercanos al sitio de Olán.

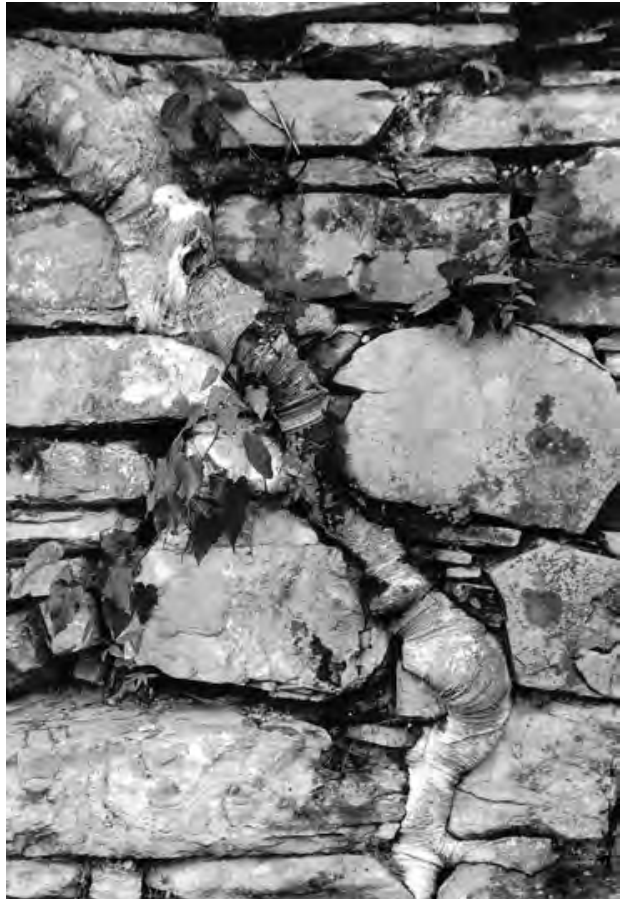

Fotografía 4. Típico deterioro de estructuras arqueológica en la zona del Plan Maestro por la vegetación y el abandono.

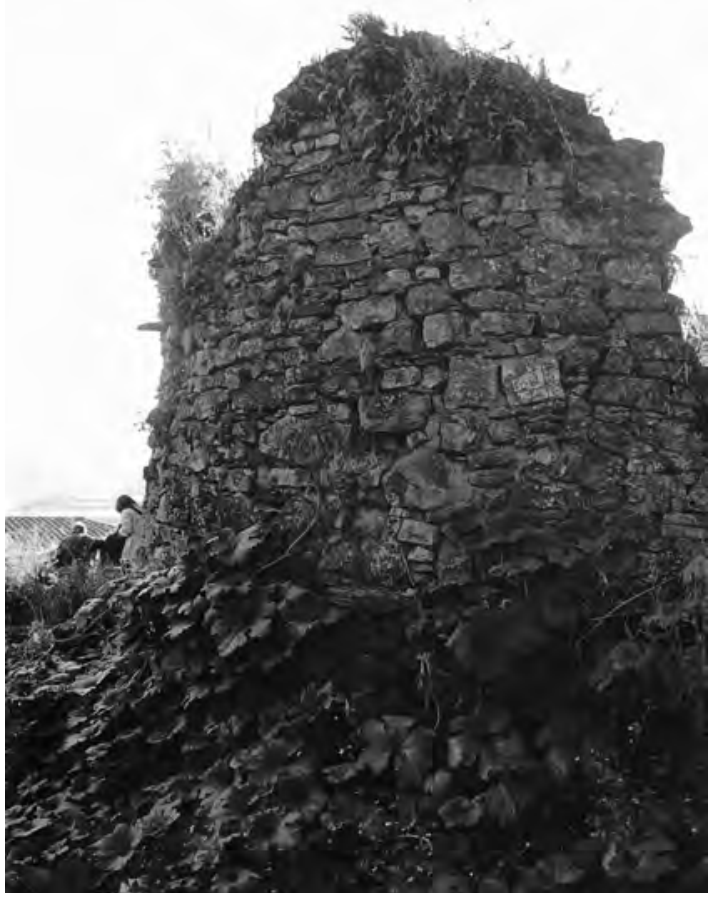

Fotografía 5. Casa Redonda, estructura circular arqueológica en el centro urbano de La Jalca, distrito de La Jalca Grande. 


\begin{tabular}{|c|c|c|c|c|c|c|c|c|c|c|c|}
\hline \multirow[t]{2}{*}{ Sitios } & \multicolumn{3}{|c|}{ Naturales } & \multicolumn{6}{|c|}{ Antrópicas } & & \multirow[t]{2}{*}{ Total } \\
\hline & $\frac{\sqrt[\widetilde{N}]{\mathbb{N}}}{\sum_{\Sigma}^{\pi}}$ & 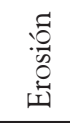 & 苞 & 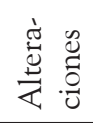 & 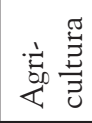 & 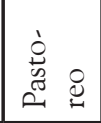 & 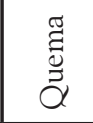 & : & 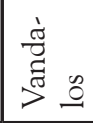 & 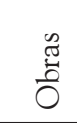 & \\
\hline Tella & $\mathrm{X}$ & $\mathrm{X}$ & $\mathrm{X}$ & & $\mathrm{X}$ & & $\mathrm{X}$ & & & & 5 \\
\hline Qhapac Ñan & - & - & - & - & - & - & - & - & - & - & - \\
\hline Yuructuna & $\mathrm{X}$ & $\mathrm{X}$ & $\mathrm{X}$ & $\mathrm{X}$ & $\mathrm{X}$ & & $\mathrm{X}$ & & & & 6 \\
\hline Pumachaca & & $\mathrm{X}$ & $\mathrm{X}$ & $\mathrm{X}$ & & & & & & $\mathrm{X}$ & 4 \\
\hline Shihual & & $\mathrm{X}$ & $\mathrm{X}$ & $\mathrm{X}$ & & & & & $\mathrm{X}$ & & 4 \\
\hline Olán & $\mathrm{X}$ & $\mathrm{X}$ & $\mathrm{X}$ & $\mathrm{X}$ & $\mathrm{X}$ & $\mathrm{X}$ & $\mathrm{X}$ & & & & 7 \\
\hline Alcalde Urco & $\mathrm{X}$ & & & & $\mathrm{X}$ & & & & & & 2 \\
\hline Revash & & $\mathrm{X}$ & $\mathrm{X}$ & $\mathrm{X}$ & & & & $\mathrm{X}$ & $\mathrm{X}$ & & 5 \\
\hline Arica/Carpona & $\mathrm{X}$ & $\mathrm{X}$ & & & $\mathrm{X}$ & & & & & & 3 \\
\hline Incapirka & $\mathrm{X}$ & $\mathrm{X}$ & & & $\mathrm{X}$ & & & & & $\mathrm{X}$ & 4 \\
\hline Monja & $X$ & $X$ & & $\mathrm{X}$ & $\mathrm{X}$ & & & & & & 4 \\
\hline Palpata & $\mathrm{X}$ & & & & & & & & & & 1 \\
\hline Shucsho & $\mathrm{X}$ & $\mathrm{X}$ & & $\mathrm{X}$ & $\mathrm{X}$ & & $\mathrm{X}$ & & & & 5 \\
\hline Carpona & & & & & & & & & & $\mathrm{X}$ & 1 \\
\hline Ollape & $\mathrm{X}$ & $\mathrm{X}$ & & $\mathrm{X}$ & $\mathrm{X}$ & $\mathrm{X}$ & $\mathrm{X}$ & & $\mathrm{X}$ & & 7 \\
\hline Casa Redonda & $\mathrm{X}$ & $\mathrm{X}$ & & $\mathrm{X}$ & & & & & & $\mathrm{X}$ & 4 \\
\hline Putquero Loma & $\mathrm{X}$ & $\mathrm{X}$ & & $\mathrm{X}$ & & & & & $X$ & & 4 \\
\hline Sec'sto & $\mathrm{X}$ & $\mathrm{X}$ & & & & & & & $\mathrm{X}$ & & 3 \\
\hline Qhapac Ñan & $\mathrm{X}$ & $\mathrm{X}$ & & $\mathrm{X}$ & $\mathrm{X}$ & & & & & $\mathrm{X}$ & 5 \\
\hline $\begin{array}{l}\text { Camino La Jalca } \\
\text { Ubillón }\end{array}$ & & $\mathrm{X}$ & & $\mathrm{X}$ & & & & & & $\bar{X}$ & 3 \\
\hline La Congona & X & $X$ & $\mathrm{X}$ & & $\mathrm{X}$ & $\mathrm{X}$ & $\mathrm{X}$ & & & & 6 \\
\hline Cataneo & $\mathrm{X}$ & $\mathrm{X}$ & $\mathrm{X}$ & $\mathrm{X}$ & $\mathrm{X}$ & & & & & & 5 \\
\hline El Molinete & $\mathrm{X}$ & $\mathrm{X}$ & & $\mathrm{X}$ & $\mathrm{X}$ & & $\mathrm{X}$ & & & & 5 \\
\hline Challuapunta & - & - & - & - & - & - & - & - & - & - & - \\
\hline Andenes & $\mathrm{X}$ & $\mathrm{X}$ & & $\mathrm{X}$ & & & & $\mathrm{X}$ & & & 4 \\
\hline Andenes & $\mathrm{X}$ & $\mathrm{X}$ & & $\mathrm{X}$ & & & & $\mathrm{X}$ & & & 4 \\
\hline SUBTOTAL & 25 & 28 & 10 & 19 & 18 & 4 & 9 & 3 & 6 & 7 & \\
\hline
\end{tabular}

\begin{tabular}{|c|c|c|c|c|c|c|c|c|c|c|}
\hline \multicolumn{11}{|c|}{ Figura 3. Presencia de problemas de primer orden de los sitios arqueológicos } \\
\hline 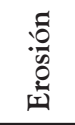 & 悹 & 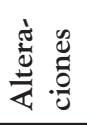 & 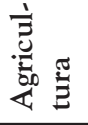 & 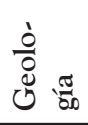 & 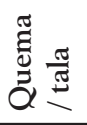 & $\frac{\mathfrak{a}}{\tilde{T}}$ & 急 & 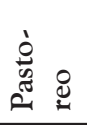 & 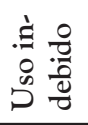 & \\
\hline 28 & 25 & 19 & 18 & 10 & 9 & 7 & 6 & 4 & 3 & № de casos \\
\hline $80 \%$ & $71 \%$ & $54 \%$ & $51 \%$ & $28 \%$ & $26 \%$ & $20 \%$ & $17 \%$ & $11 \%$ & $8 \%$ & $\%$ \\
\hline
\end{tabular}




\subsection{Problemas de Segundo Orden}

\begin{tabular}{|l|l|}
\hline \multicolumn{1}{|c|}{ Figura 4. El INC } \\
\hline \multicolumn{1}{|c|}{ Problema } & \multicolumn{1}{c|}{ Causa } \\
\hline $\begin{array}{l}\text { 1. Deterioro y destrucción progresiva de los sitios arqueo- } \\
\text { lógicos: abandono }\end{array}$ & Reducida inversión y vigilancia \\
\hline 2. Debilidad de defensa legal y administrativa de los sitios & Indefinición legal de los sitios \\
\hline $\begin{array}{l}\text { 3. Débil protección de los sitios y de las áreas de reserva y } \\
\text { paisajísticas }\end{array}$ & Alianzas débiles \\
\hline 4. Intervenciones inadecuadas, limitadas o tardías & $\begin{array}{l}\text { Limitados recursos humanos, técnicos y } \\
\text { materiales }\end{array}$ \\
\hline 5. Conductas negativas y desinformación & Pocas acciones de promoción y difusión \\
\hline
\end{tabular}

Figura 5. Las municipalidades

\begin{tabular}{|l|l|}
\hline \multicolumn{1}{|c|}{ Problema } & \multicolumn{1}{c|}{ Causa } \\
\hline $\begin{array}{l}\text { 1. No hay planes de manejo municipal de los sitios } \\
\text { arqueológicos }\end{array}$ & $\begin{array}{l}\text { No hay un presupuesto estable para la conserva- } \\
\text { ción del patrimonio arqueológico }\end{array}$ \\
\hline 2. Imposibilidad de realizar limpiezas & $\begin{array}{l}\text { Fondos insuficientes para el pago de especialistas } \\
\text { permanentes }\end{array}$ \\
\hline $\begin{array}{l}\text { 3. Restos arqueológicos urbanos deteriorados sin } \\
\text { intervención municipal }\end{array}$ & $\begin{array}{l}\text { Débil capacidad técnica y administrativa de la } \\
\text { municipalidad para cumplir su función }\end{array}$ \\
\hline
\end{tabular}

\begin{tabular}{|l|l|}
\hline \multicolumn{2}{|c|}{ Figura 6. Debilidades educativas } \\
\hline \multicolumn{1}{|c|}{ Problema } & \multicolumn{1}{c|}{ Causa } \\
\hline 1. Actos de vandalismo, saqueo y destrucción & Débil valoración cultural y desconocimiento \\
\hline 2. Uso no educativo del monumento arqueológico & $\begin{array}{l}\text { Débil inclusión en los programas y currículo del } \\
\text { patrimonio arqueológico }\end{array}$ \\
\hline $\begin{array}{l}\text { (La escuela alejada del patrimonio arqueológico y } \\
\text { cultural de la región) }\end{array}$ & $\begin{array}{l}\text { Materiales educativos no incluyen los monumentos } \\
\text { arqueológicos locales }\end{array}$ \\
\hline & $\begin{array}{l}\text { Bajo acceso de los docentes a bibliografía especiali- } \\
\text { zada y a contacto con especialistas de arqueología }\end{array}$ \\
\hline
\end{tabular}

\begin{tabular}{|l|l|}
\hline \multicolumn{2}{|c|}{ Figura 7. La investigación arqueológica } \\
\hline \multicolumn{1}{|c|}{ Problema } & \multicolumn{1}{c|}{ Causa } \\
\hline $\begin{array}{l}\text { Vacíos de investigación y conocimiento del } \\
\text { significado de los monumentos }\end{array}$ & $\begin{array}{l}\text { Investigaciones (excavaciones y análisis) arqueológicas } \\
\text { previas y actuales escasas }\end{array}$ \\
\hline Escasos arqueólogos trabajando en la zona & Poca especialización de los trabajos existentes \\
\hline Alto costo de investigaciones & Recursos instrumentales especializados para análisis \\
\hline
\end{tabular}




\section{Calificación de los Sitios ARQueológicos}

\subsection{Indicadores de Potencial de los Sitios Arqueológicos}

La "calificación" es la selección de los sitios arqueológicos a partir de la valoración de su vocación para la realización de diversos tipos de proyectos y acciones acordes al cronograma, metas y productos definidos por el Plan Maestro. Para ello, se establecieron una serie de parámetros de observación que permitieran definir aquellos sitios que por su potencial y problemática, debían ser priorizados en los planes de intervención. Del mismo modo, se determinaron las formas y diseños de adecuación a nuevas funciones sociales y a los servicios requeridos para la puesta en uso social de los sitios.

\subsubsection{Parámetros de la calificación}

Los indicadores del potencial de los sitios para las intervenciones de investigación, conservación, y acondicionamiento fueron los siguientes: facilidad de acceso, necesidad de intervenciones de conservación/investigación urgentes, importancia del sitio arqueológico, cercanía a centros poblados y la cercanía a otros atractivos turísticos y culturales.

Para medir los indicadores se establecieron valores numéricos del 0 al 9, con 5 grados distintos de escala: Muy Alta $(8,9)$, Alta $(6,7)$, Media (5), Baja $(3,4)$, Muy baja (2) y Desconocida (0) (Figura 8). Se trataba de valores referenciales y relativos que solo pueden ser especificados mediante un mayor conocimiento de los sitios pero que, sin embargo, sirvieron para tener una mejor idea del conjunto.

A continuación se describen cada uno de los parámetros usados:

\section{- La facilidad del acceso}

Se calificó la posibilidad de acceso al sitio por distintos tipos de público. El acceso es condicionante de la capacidad de movilización de los agentes locales para las tareas de difusión, promoción, vigilancia y protección así como para la realización de proyectos de investigación y conservación. Sirve también para especificar los grupos de visitantes según su capacidad física y equipo, la duración de las visitas y el nivel de intervención necesario para la adecuación de éstos al turismo. Se consideraron los caminos de acceso a los sitios desde el punto más cercano de la carretera o pueblos hasta estos.

Los elementos de evaluación fueron el tiempo, la distancia, los grados de pendiente, el estado del camino y los riesgos.

\section{- La necesidad de intervenciones urgentes de conservación}

El reconocimiento de campo y la información de los sitios registrados, permitieron un acercamiento superficial a la problemática de la conservación de estructuras arqueológicas. Sin embargo, el mal estado para su aprovechamiento científico, educativo y turístico, se hizo evidente. Todos los sitios tuvieron una Muy Alta (MA) necesidad de realización de trabajos de conservación y en este aspecto han sido calificados con el máximo puntaje (9). Las mayores necesidades identificadas fueron la limpieza de malezas y escombros, reposición de elementos constructivos, consolidación de estructuras arquitectónicas, conservación de enlucidos y otras actividades referidas a la conservación.

Es importante considerar que las intervenciones de conservación deben ser siempre acompañadas de excavaciones arqueológicas en los procesos de evaluación y liberación de estructuras. Además, se incluye también la necesidad de recuperar material arqueológico mediante excavaciones para su análisis, interpretación y reconstrucciones históricas, como tarea fundamental para cualquier actividad expositiva que se pretendiera realizar. Iguales criterios se aplican para las colecciones de material arqueológico. Igualmente, las intervenciones debían incluir acciones de negociación con los agricultores u otros, que realizan cultivos, pastoreo o actividades diversas en las áreas arqueológicas (Olán, Ollape, Tella, Cataneo, Molinete y Yuructuna) a fin de detener estos actos inmediatamente. 


\section{- La cercanía a centros poblados}

Esta variable se incluyó por considerarse como un importante efecto esperado del PMK el uso continuado e intenso de los sitios por los pobladores cercanos, permitiéndose su fácil integración a los programas que las escuelas de los alrededores, las municipalidades distritales, los movimientos vecinales y las comunidades puedan realizar. Por esa misma razón, la adecuación de espacios y la previsión de riesgos y accidentes de los visitantes así como la preparación del sitio para recibir continuamente a los visitantes, eran una prioridad a tomar en cuenta en este tipo de sitios.

Los elementos de evaluación fueron el tiempo y la distancia entre el centro poblado y el sitio arqueológico. La calificación más alta la tiene la Casa Redonda, ubicada en el mismo centro poblado de La Jalca, mientras los más lejanos son Tella, Shihual (en relación a Magdalena) y Shucsho en (relación a Montevideo).

\section{- La importancia de los sitios}

Se consideró la extensión del sitio, el número y tipos de estructuras, los elementos decorativos (tipo, cantidad, singularidad), por su significado arqueológico y por su potencial para la adecuación de espacios para servicios diversos. El potencial paisajístico y visual fue también importante en la calificación de los sitios más aun por la presencia en el Utcubamba de muchos sitios en las partes altas de las montañas desde donde se tiene vistas espectaculares del valle del Utcubamba.

\section{- La cercanía a otros atractivos}

Se mide las posibilidades del sitio arqueológico a integrarse a circuitos de visita que incluyeran más sitios arqueológicos o atractivos naturales. Se evaluaron los elementos siguientes: distancia entre los atractivos, la diversidad, la complementariedad, su importancia, la integralidad como parte de un paisaje arqueológico especialmente para los casos de andenes y terrazas de cultivo.

Un caso importante es el de Kuélap. Éste tie- ne una valoración alta pues se encuentran muy cerca varios sitios arqueológicos como la muralla de San José, las terrazas de cultivos de El Lirio y el poblado de Imperio. La presencia de estos sitios le da un carácter de integridad como paisaje cultural arqueológico.

Los resultados de la calificación de necesidades de intervención se muestran en las Figuras 8,9 y 10.

\begin{tabular}{|l|c|}
\hline \multicolumn{2}{|c|}{ Figura 8. Escala de valores } \\
\hline \multicolumn{1}{|c|}{ Escala (0 al 9) } & Valores \\
\hline $\mathrm{MA}=$ muy alta & 9 \\
\hline $\mathrm{A}=$ alta & 7 \\
\hline $\mathrm{M}=$ media & 5 \\
\hline $\mathrm{B}=$ baja & 3 \\
\hline $\mathrm{MB}=$ muy baja & 1 \\
\hline $\mathrm{D}=$ desconocida & 0 \\
\hline
\end{tabular}

\subsubsection{Interpretación de los resultados}

La poca información para varios de los sitios, hizo que los indicadores sean provisionales hasta la realización de evaluaciones pormenorizadas. Sin embargo, se realizó la calificación con los datos disponibles en términos de establecer una metodología aplicable a este tipo de proyectos.

\section{- Prioridades por tipo de indicador}

El primer indicador, el de puntaje acumulado más alto, es el relativo a la necesidad de llevar a cabo intervenciones urgentes en los sitios arqueológicos (239 puntos sobre 28 Sitios con Información - SCI). El mal estado de conservación era el principal factor limitante para el desarrollo del PMK. En el mismo nivel de importancia, pero como factor causal, está el tema de la indefinición legal de los sitios arqueológicos cuya solución requería de la realización de expedientes técnicos.

La segunda calificación, la cercanía entre diversos tipos de atractivos turísticos (161 puntos sobre $25 \mathrm{SCI}$ ) reflejaba el potencial del conjunto del área para la realización de circuitos educativos o turísticos que incorporen distintos tipos de 


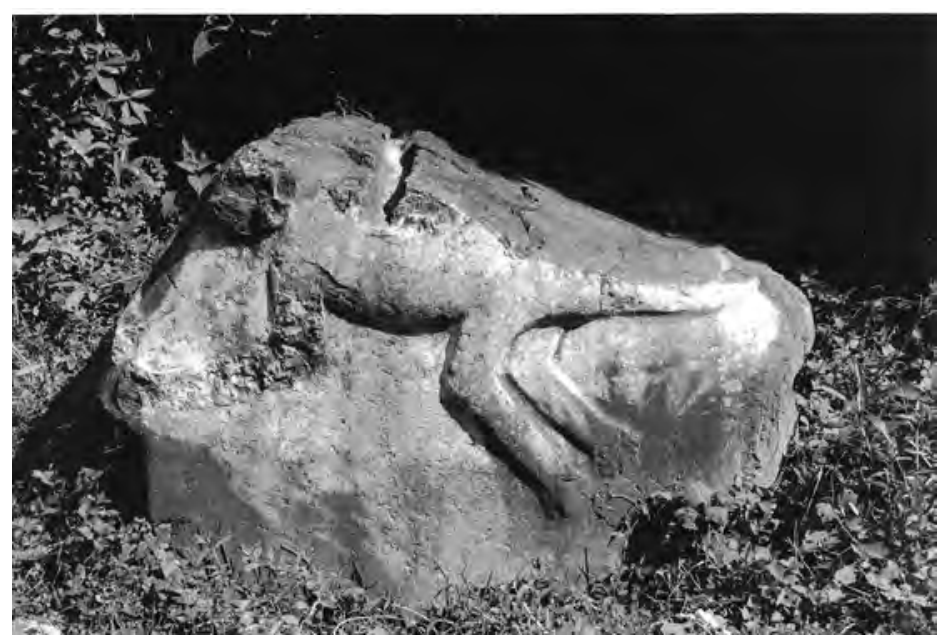

Fotografía 6. Pumachaca, piedra tallada al borde de carretera, en la Unidad Geohistórica Ribera del Utcubamba.

Fotografía 7. Ollape, Distrito de la Jalca Grande, estructuras arqueológicas alteradas por intervenciones contemporáneas.
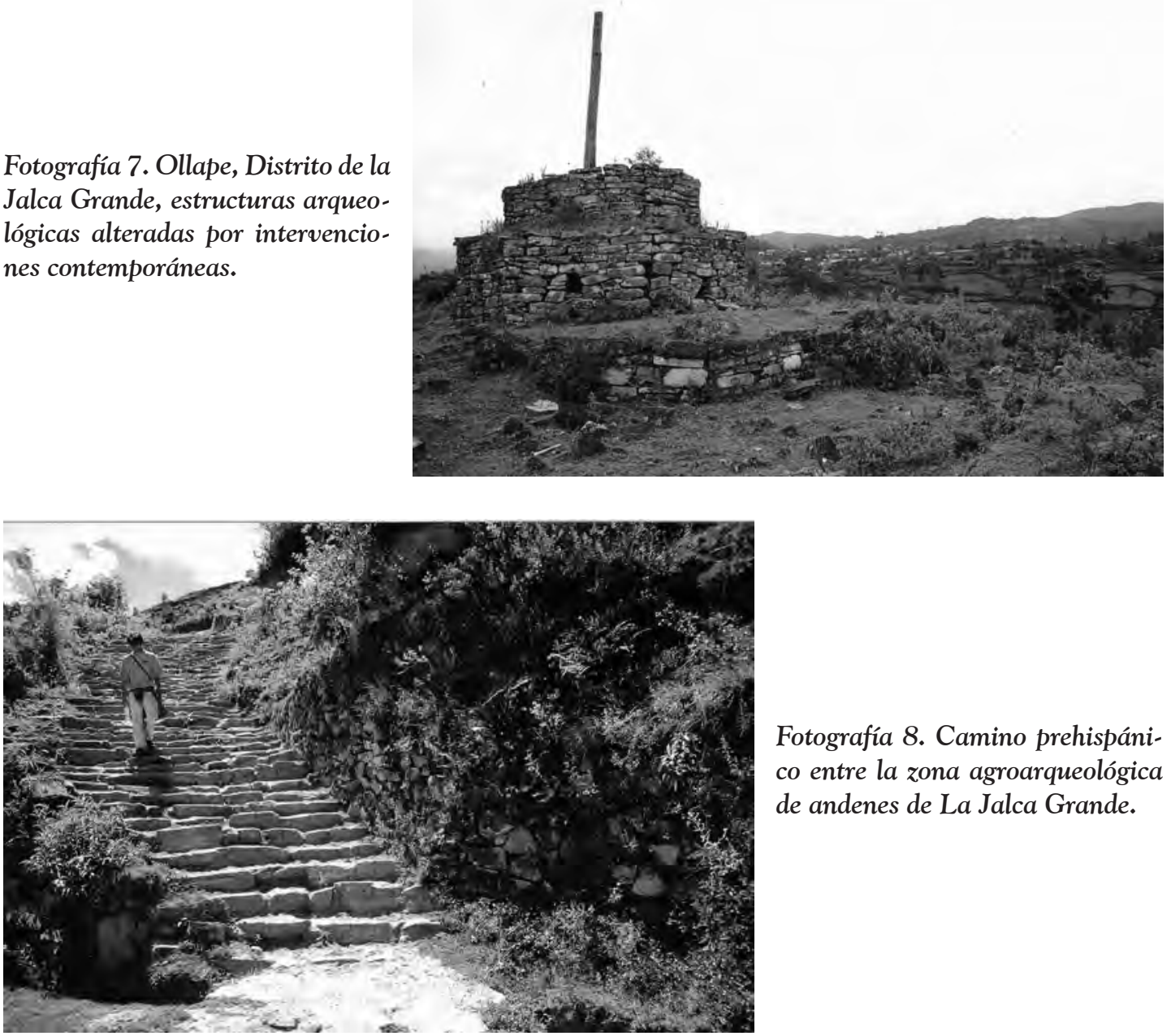

Fotografía 8. Camino prehispánico entre la zona agroarqueológica de andenes de La Jalca Grande. 
Figura 9. Valoración de indicadores por sitio

\begin{tabular}{|c|c|c|c|c|c|c|}
\hline Distrito/Sitio & $\begin{array}{c}\text { Facilidad } \\
\text { de acceso }\end{array}$ & $\begin{array}{c}\text { Intervención } \\
\text { urgente }\end{array}$ & $\begin{array}{c}\text { Importan- } \\
\text { cia }\end{array}$ & $\begin{array}{c}\text { Cercanía a } \\
\text { poblado }\end{array}$ & $\begin{array}{c}\text { Atractivos } \\
\text { cercanos }\end{array}$ & TOTAL \\
\hline
\end{tabular}

Tingo

\begin{tabular}{|l|l|l|l|l|l|l|}
\hline 1. Kuelap & 9 & 9 & 9 & 7 & 9 & 41 \\
\hline 2. Tuich/ Silic & 7 & 9 & 5 & 7 & 7 & 35 \\
\hline 3. San José & 5 & 9 & 7 & 5 & 7 & 33 \\
\hline 4. Lirio & 5 & 9 & 7 & 5 & 7 & 33 \\
\hline 5. Imperio & 5 & 9 & 5 & 5 & 7 & 31 \\
\hline 6. Américas & 0 & 0 & 0 & 0 & 0 & 0 \\
\hline 7. Santa Clara & 0 & 0 & 0 & 0 & 0 & 0 \\
\hline
\end{tabular}

La Jalca Grande

\begin{tabular}{|l|l|l|l|l|l|l|}
\hline 8. Casa Redonda & 9 & 9 & 7 & 7 & 7 & 39 \\
\hline 9. Ollape & 7 & 9 & 7 & 7 & 7 & 37 \\
\hline 10. Camino Inca & 9 & 9 & 7 & 5 & 7 & 35 \\
\hline 11. Cam.Prehispán. & 9 & 9 & 7 & 5 & 7 & 35 \\
\hline 12. Área andenes & 9 & 7 & 7 & 5 & 7 & 35 \\
\hline 13. Putquero Loma & 0 & 0 & 0 & 0 & 0 & 0 \\
\hline 14. Sec'sto & 0 & 0 & 0 & 0 & 0 & 0 \\
\hline
\end{tabular}

Mariscal Castilla

\begin{tabular}{|l|l|l|l|l|l|l|}
\hline 15. Olán & 5 & 9 & 9 & 7 & 7 & 37 \\
\hline 16. Área andenes & 5 & 7 & 7 & 7 & 7 & 33 \\
\hline 17. Alcalde Urco & 0 & 9 & 0 & 0 & 7 & 16 \\
\hline
\end{tabular}

Santo Tomás

\begin{tabular}{|l|l|l|l|l|l|l|}
\hline 18. Revash & 5 & 9 & 9 & 5 & 9 & 37 \\
\hline
\end{tabular}

Magdalena

\begin{tabular}{|l|l|l|l|l|l|l|}
\hline 19. Macro & 5 & 9 & 7 & 5 & 7 & 33 \\
\hline 20. Pumachaca & 9 & 9 & 5 & 5 & 5 & 33 \\
\hline 21. Yuructuna & 5 & 9 & 5 & 5 & 5 & 29 \\
\hline 22. Machupirca & 5 & 9 & 5 & 5 & 5 & 29 \\
\hline 23. Tella & 3 & 9 & 7 & 3 & 5 & 27 \\
\hline 24. Shihual & 1 & 9 & 3 & 3 & 3 & 19 \\
\hline 25. Camino Inca & 0 & 0 & 0 & 0 & 0 & 0 \\
\hline Montevideo & 7 & 9 & 7 & 5 & 5 & 33 \\
\hline 26. Ingapirca & 1 & 9 & 5 & 3 & 3 & 21 \\
\hline 27. Shucsho & \multicolumn{7}{|l|}{}
\end{tabular}




\begin{tabular}{|l|c|c|c|c|c|c|}
\hline \multicolumn{1}{|c|}{ Distrito/Sitio } & $\begin{array}{c}\text { Facilidad de } \\
\text { acceso }\end{array}$ & $\begin{array}{c}\text { Intervención } \\
\text { urgente }\end{array}$ & $\begin{array}{c}\text { Importan- } \\
\text { cia }\end{array}$ & $\begin{array}{c}\text { Cercanía a } \\
\text { poblado }\end{array}$ & $\begin{array}{c}\text { Atractivos } \\
\text { cercanos }\end{array}$ & TOTAL \\
\hline 28. Carpona & 0 & 0 & 0 & 0 & 0 & 0 \\
\hline 29. Palpata & 0 & 0 & 0 & 0 & 0 & 0 \\
\hline 30. Arica & 0 & 9 & 0 & 0 & 0 & 0 \\
\hline 31. Monja & 0 & 0 & 0 & 0 & 0 & 0 \\
\hline S. F. Del Yeso & \multicolumn{7}{|l|}{} & & & \\
\hline 32. La Congona & 3 & 9 & 5 & 3 & 7 & 27 \\
\hline 33. Cataneo & 1 & 9 & 5 & 3 & 7 & 25 \\
\hline 34. El Molinete & 1 & 9 & 5 & 3 & 7 & 25 \\
\hline 35. Challuapunta & 0 & 0 & 0 & 0 & 0 & 0 \\
\hline TOTAL & 130 & 239 & 152 & 120 & 161 & \\
\hline
\end{tabular}

Indicadores de potencial por sitio arqueológico

Total por sitio

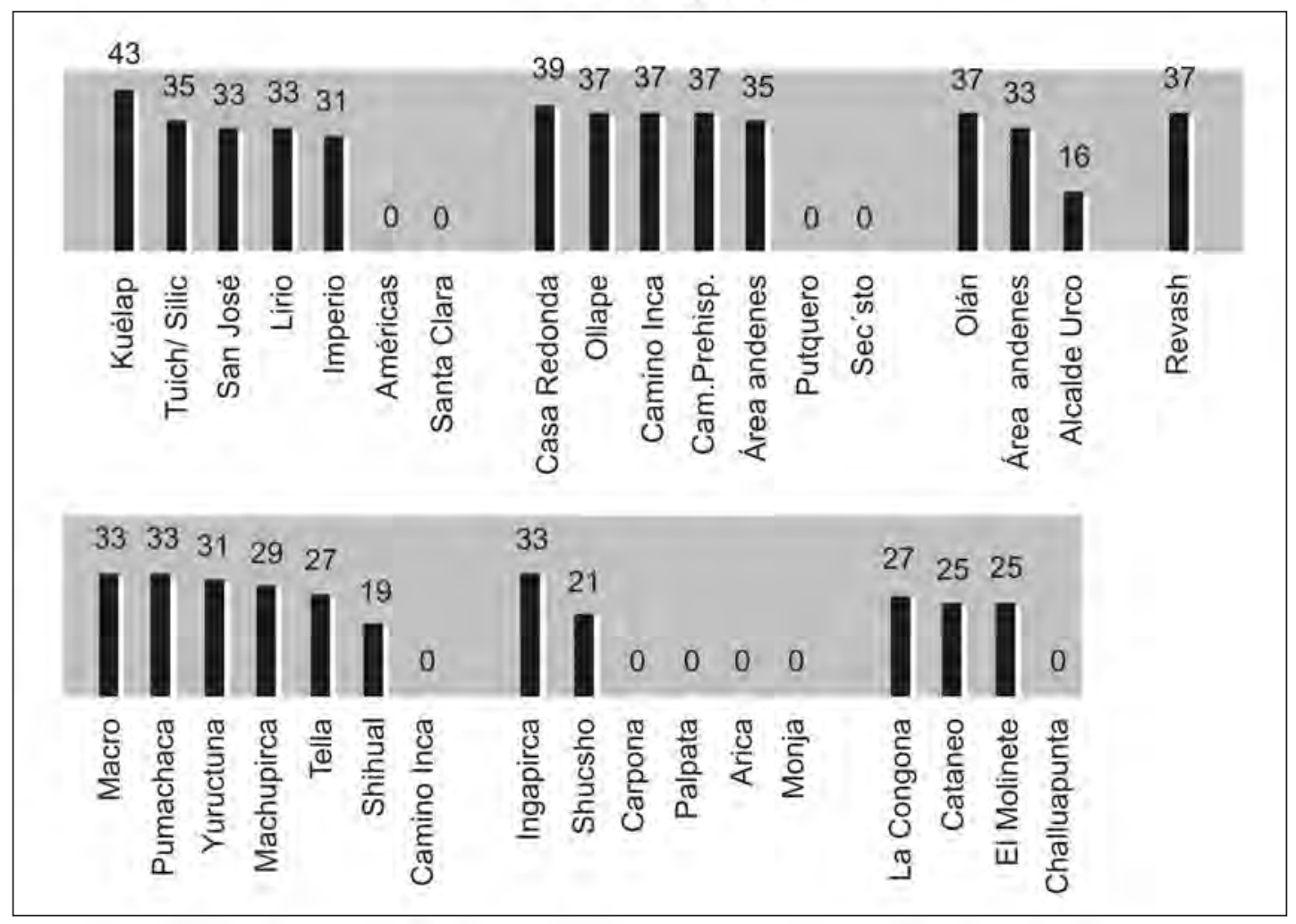

Figura 10. Indicadores de potencial por sitio arqueológico (barras) 
atractivos (arqueológicos, naturales, culturales) o a los sitios arqueológicos entre sí.

La tercera calificación la obtuvo la importancia arqueológica de los sitios (152 puntos sobre $24 \mathrm{SCI}$ ). Sin embargo, el puntaje de este indicador es el reflejo directo de la falta de información para los sitios. Las visitas de campo permitieron observar que los asentamientos arqueológicos se caracterizaban en su mayoría por ser manifestaciones arquitectónicas importantes. Por otro lado, los sitios arqueológicos aumentará su importancia en la medida que sean más conservados e investigados.

La cuarta calificación la obtuvo la facilidad de los accesos (130 puntos sobre 24 SCI). Esto se explica debido a que pese al potencial de los sitios para vincularse, principalmente por las distancias relativamente cortas, la dificultad facilidad de los accesos alcanza un punto medio, insuficiente para integrar los diversos atractivos en circuitos de visita, así como para otras tareas relativas al PMK.

En el último lugar de valoración aparecía la cercanía a centros poblados (120 puntos sobre $24 \mathrm{SCI})$. En este caso el puntaje obtenido es debido principalmente a que el área del PMK se caracteriza por un poblamiento de tipo rural.

\section{- Priorización por sitios}

Se estableció que los sitios de mayor potencial eran los que se encontraban en un rango de clasificación Muy alto $(8,9)$. El sitio de mayor calificación tanto por sus potenciales como por sus factores limitantes es el de Kuélap con 41 puntos. Se trata del sitio eje de todo el PMK, con investigaciones e intervenciones previas y es y ha sido siempre un polo de atracción regional. No es de extrañar esta calificación.

En segundo lugar está la Casa Redonda de la Jalca Grande con 39 puntos. Este puntaje se basaba principalmente en su ubicación en el mismo centro poblado lo que le daba condiciones de acceso muy altas y al mismo tiempo por su cercanía a otros atractivos (museo de La Jalca, la Iglesia colonial de la plaza, sus costumbres, tra- diciones). Además, su importancia arqueológica por ser uno de los pocos sitios que cuenta con información gráfica, le daban un gran potencial a este sitio para actividades expositivas.

Ollape, Olán y Revash, constituían un grupo homogéneo en potencial para su intervención por el Plan Maestro (37). Se trata de sitios que por la importancia de sus restos arquitectónicos debieron haber tenido una importancia especial en la época prehispánica. Esto se complementaba por el hecho de tener un acceso relativamente sencillo (especialmente los dos primeros), otros atractivos arqueológicos y poblados cercanos y, en el caso de Revash, por corresponder a un sitio funerario distinto a la mayoría de restos arqueológicos del área.

En cuarto lugar, se ubicaron los caminos incaicos o preincaicos y las áreas agroarqueológicas. Estas últimas predominan en el paisaje y están asociadas tanto a Olán como a Ollape y a Kuélap (El Lirio, San José). El puntaje, que varía del 33 al 37 (el puntaje de 33 se debía más a la falta de referencias y de visita a algunos de estos lugares), permitía considerar a estos sitios como un grupo homogéneo y en el mismo nivel los anteriores. Es interesante que la oferta temática en estos casos sea también complementaria y plantea una posibilidad distinta a la abundancia de asentamientos arqueológicos que siguen un mismo patrón constructivo.

En quinto lugar, aparecía el grupo de aldeas arqueológicas de menor jerarquía que las anteriormente mencionadas alcanzando un puntaje menor $(31$ - 33). En esta calificación se incluyen Macro, Yuructuna, Shucsho, Imperio, Pumachaca e Ingapirka. En los dos últimos casos el valor principal reside en su cercanía a la carretera y en corresponder a dos manifestaciones poco presentes en el área (arquitectura inca, escultura en piedra respectivamente.

En sexto lugar, un grupo de sitios de puntajes más bajos (25 - 27); forman este grupo Machupirca, Tella, la Congona, Cataneo y Molinete. Son de menor jerarquía arquitectónica y extensión. Igualmente presentaban una mayor 
lejanía de los centros poblados y dificultad de acceso. A excepción de la Congona, que presenta una importancia arquitectónica mayor que la del resto, los otros sitios pueden ser considerados como "reserva" para la investigación científica. Tella es un gran sitio arqueológico, sin embargo, las calificaciones baja en los otros aspectos desplazan a este a este grupo. Su intervención debe ser evaluada en función al circuito de sitios del distrito de Magdalena.

Como séptimo grupo quedaron los sitios de Alcalde Urco, Carpona, y Shihual. Los dos primeros deben aún ser visitados y en el caso de Shihual, no presenta gran encanto arqueológico pero sí interés espeleológico, natural y paisajístico (entierros saqueados al interior de caverna natural con estalactitas). Shihual debía considerarse para reserva de investigaciones especializadas, igualmente en el caso de Carpona. Sabemos por referencias que se trataba del lugar de enterramiento de los habitantes de la aldea arqueológica de Shucsho y que es una caverna natural como la de Shihual.

\section{- Priorización de sitios por distritos}

Los sitios de mayor puntaje se reparten homogéneamente entre todos los distritos a excepción de los distritos de Leymebamba y San Juan de Lopecancha, los cuales no cuentan con sitios arqueológicos registrados al interior del área del PMK (en Leymebamba se encuentra el Museo con los materiales de la Laguna de los Cóndores).

Las cabezas de serie según los puntajes obtenidos son Kuélap en el distrito de Tingo, Casa Redonda en Jalca Grande, Olán en Mariscal Castilla (involucra a Montevideo por la corta distancia), Revash en Santo Tomás, Ingapirka en Montevideo y Macro en el distrito de Magdalena (se puede incluir con Pumachaca por su ubicación al borde de la carretera y su carácter de resto arqueológico aislado) y La Congona en el distrito de San Francisco del Yeso. Esta dis- tribución de sitios importantes en cada distrito, es un potencial importante pues permite pensar en intervenciones arqueológicas equilibradas en cada uno de ellos, de modo que se eviten "recelos" entre los municipios involucrados. Por otro lado, cada municipio puede gestionar una Unidad Geohistórica ${ }^{4}$ - UGH (a excepción de la UGH. de Olán).

Los distritos con mayor cantidad de sitios arqueológicos son Tingo y La Jalca Grande con un puntaje de 7, Montevideo con 6, San Francisco del Yeso con 4, Mariscal Castilla con 3 y Santo Tomás con 1(incluye gran cantidad de mausoleos en farallones rocosos aun por registrar).

\subsection{Priorización de actividades por cada sitio}

A partir del diagnóstico de conservación se detectaron las actividades necesarias y prioritarias a desarrollar en el conjunto de sitios. El orden obtenido de la valoración de los indicadores de potencial y limitaciones (las escalas y valores usadas son las mismas en ambos casos), no necesariamente corresponden con el de las actividades. Esto se debe a que la valoración acá representa el peso específico o grado de intensidad necesario de las intervenciones en cada uno de los sitios.

Las actividades incluidas en esta definición incluían: conservación de estructuras arquitectónicas, conservación de colecciones arqueológicas, investigación arqueológica, mejoramiento o habilitación de áreas de servicio y visita, mejoramiento de sistemas de transporte vehicular, mejoramiento de caminos de herradura, integración del monumento a la escuela, gestión comunal (mantenimiento, vigilancia, protección, actividades productivas), intervención en sitios arqueológicos cercanos y, por último, actividades de promoción y difusión (Figura 11).

Del total de los 35 sitios comprendidos en el plan, se incluyeron en esta priorización los 15 sitios que contaban con información suficiente y con la calificación más alta en los indicadores de

4 Las Unidades Geohistóricas se determinaron en reuniones del equipo del PMK. Éstas son unidades territoriales en las cuales convergen rasgos geográficos, históricos y culturales dándole una unidad diferenciada de otras. 


\begin{tabular}{|l|}
\hline \multicolumn{1}{|c|}{ Figura 11. Actividades de intervención } \\
\hline 1. Conservación de estructuras arquitectónicas \\
\hline 2. Actividades de promoción y difusión \\
\hline 3. Investigación arqueológica \\
\hline 4. Intervención en sitios arqueológicos cercanos \\
\hline 5. Mejoramiento de caminos de herradura \\
\hline 6. Gestión comunal (mantenimiento, vigilancia, protección, actividades productivas) \\
\hline 7. Mejoramiento o habilitación de áreas de servicio y visita \\
\hline 8. Integración del monumento a la escuela \\
\hline 9. Mejoramiento de sistemas de transporte vehicular \\
\hline 10. Conservación de colecciones arqueológicas \\
\hline
\end{tabular}

potencial. Los sitios incluidos son los siguientes: Kuélap, Revash, Ollape, Olán, Casa Redonda, La Congona, Tella, Ingapirka, Tuich/Silic, Yuructuna, Macro, Machupirca, Cataneo, El Molinete y Pumachaca. Los resultados de la calificación se encuentran expresados más abajo (Figura 12) y ordenados según la valoración de actividades por sitio (Figura 13) y la valoración general por tipo de actividad (Figura 14).

\subsubsection{Análisis de actividades potenciales}

En función a las tablas de valoración anteriores (figuras 11, 1213,14 ) y a su distribución en el conjunto de sitios, la primera prioridad se centró en las intervenciones de conservación e investigación de los restos arqueológicos con una valoración muy alta (135 puntos) y distanciada del resto de actividades. Se trataba de detener el proceso de deterioro de manera inmediata de ahí que le fueran asignados los mayores puntajes. Un segundo grupo de alta valoración lo constituyen las actividades de promoción y difusión (97 puntos) junto a las de investigación arqueológica (93 puntos). Esto se relacionaba con generar conocimientos para los visitantes y elaborar los expedientes técnicos para la Declaración de Patrimonio Arqueológico del INC. El tercer nivel de valoración se conformó por la necesidad de Intervenciones Arqueológicas (83 puntos) en sitios cercanos entre ellos para el establecimiento de microcircuitos de visita.
Se trataba de manejar a los sitios integrados en circuitos que sustenten la Declaración de áreas de Paisaje Cultural Arqueológico por el INC. En cuarto lugar y en un rango intermedio con las prioridades anteriores, está el mejoramiento de los caminos de herradura (78 puntos) y la gestión comunal (mantenimiento, vigilancia, protección, actividades productivas) (74 puntos) mediante el acondicionamiento de los caminos a los sitios y la habilitación de áreas de actividad y descanso con fines educativos y recreativos.

Otro grupo de actividades que se referían al equipamiento para la puesta en uso social de los sitios se valoraron de la siguiente manera. El mejoramiento o habilitación de áreas de servicio para la visita (70 puntos), se combina también con la gestión comunal en el aprovechamiento de los recursos arqueológicos (74 puntos). En estos casos se trataba de la combinación de la realización de obras de acondicionamiento con la participación de los agentes locales. En quinto lugar de valoración se ubicó el uso del monumento arqueológico como instrumento educativo (60 puntos). Esta baja valoración se debía a que no todos los sitios presentaban condiciones ni espacios adecuados para su uso por grupos de escolares. Sin embargo, esto no resta la importancia del aprovechamiento educativo de los monumentos arqueológicos en los que esta actividad es posible. La sexta valoración fue para los sistemas de transporte vehicular (53 pun- 


\begin{tabular}{|l|c|c|c|c|c|c|c|c|c|c|c|}
\hline \multirow{2}{*}{ Sitio } & \multicolumn{7}{|c|}{ Figura 12. Valoración de actividades de intervención* } & \multirow{2}{*}{ TOTAL } \\
\cline { 2 - 12 } & 1 & 2 & 3 & 4 & 5 & 6 & 7 & 8 & 9 & 10 & \\
\hline 1. Kuélap & 9 & 9 & 9 & 9 & 9 & 9 & 9 & 9 & 9 & 9 & 90 \\
\hline 2. Revash & 9 & 9 & 6 & 3 & 9 & 5 & 9 & 1 & 9 & 9 & 69 \\
\hline 3. Olán & 9 & 9 & 6 & 0 & 9 & 9 & 6 & 9 & 9 & 0 & 66 \\
\hline 4. Casa Redonda & 9 & 9 & 6 & 9 & 0 & 9 & 5 & 9 & 0 & 9 & 65 \\
\hline 5. Ollape & 9 & 9 & 6 & 5 & 1 & 9 & 5 & 9 & 5 & 3 & 61 \\
\hline 6. La Congona & 9 & 9 & 6 & 6 & 6 & 5 & 5 & 5 & 5 & 0 & 56 \\
\hline 7. Tella & 9 & 5 & 6 & 6 & 6 & 5 & 5 & 3 & 5 & 0 & 50 \\
\hline 8. Ingapirca & 9 & 6 & 6 & 3 & 9 & 3 & 5 & 3 & 1 & 0 & 45 \\
\hline 9. Tuich/Silic & 9 & 6 & 6 & 6 & 1 & 5 & 3 & 9 & 0 & 0 & 45 \\
\hline 10. Yuructuna & 9 & 3 & 6 & 6 & 6 & 5 & 3 & 3 & 0 & 0 & 41 \\
\hline 11. Macro & 9 & 6 & 6 & 6 & 6 & 5 & 3 & 0 & 0 & 0 & 41 \\
\hline 12. Machupirca & 9 & 5 & 6 & 6 & 6 & 5 & 1 & 0 & 0 & 0 & 38 \\
\hline 13. Cataneo & 9 & 3 & 6 & 6 & 5 & 0 & 3 & 0 & 5 & 0 & 38 \\
\hline 14. El Molinete & 9 & 3 & 6 & 6 & 5 & 0 & 3 & 0 & 5 & 0 & 38 \\
\hline 15. Pumachaca & 9 & 6 & 6 & 6 & 0 & 0 & 5 & 0 & 0 & 0 & 32 \\
\hline TOTAL & 135 & 97 & 93 & 83 & 78 & 74 & 70 & 60 & 53 & 30 & \\
\hline * Los números en la fila de encabezamiento corresponden a la actividad numerada en la Figura 11. \\
\hline
\end{tabular}

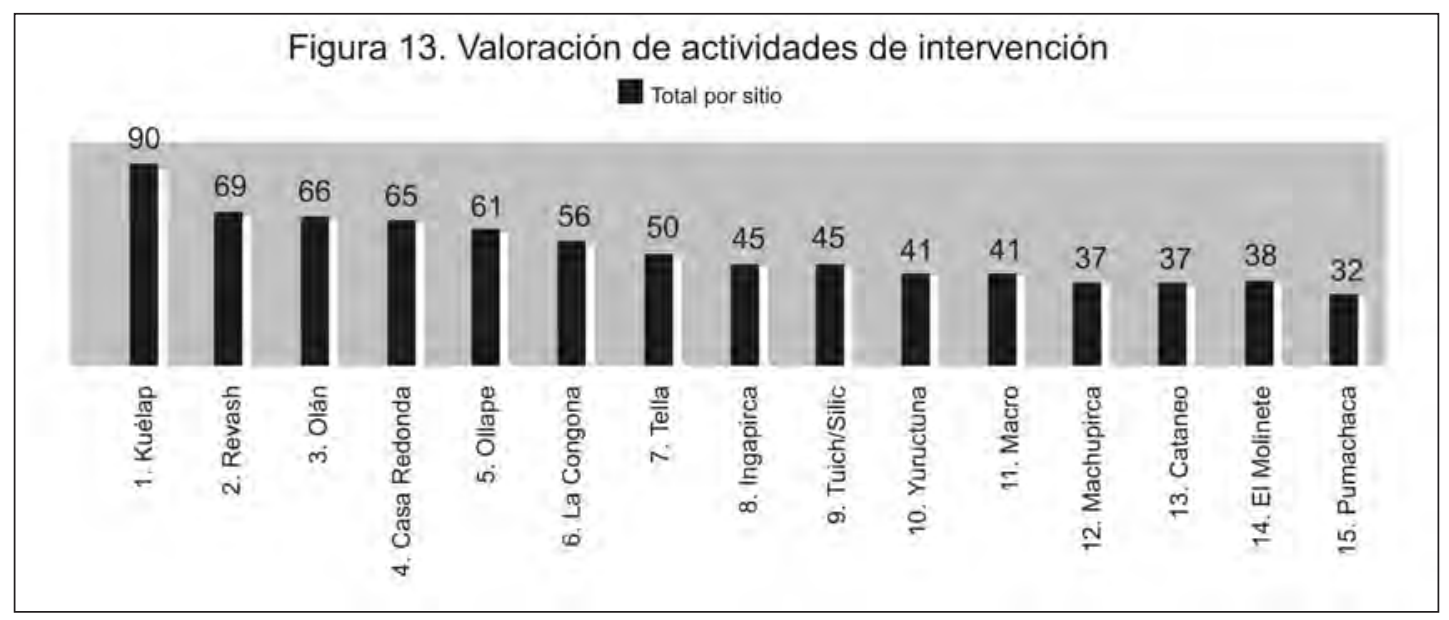

Figura 13. Valoración de actividades por sitio. 


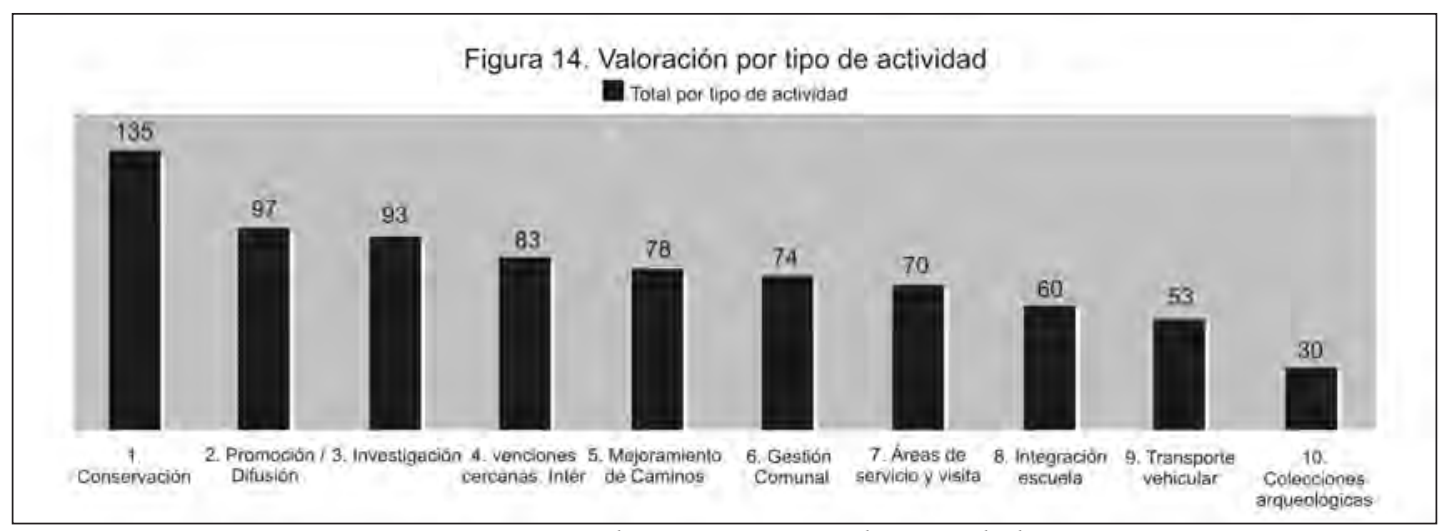

Figura 14. Valoración por tipo de actividad.

tos). Consiste en la regularización de servicios de transporte, especialmente para acceder desde los centros poblados que cuentan normalmente con servicio público hacia las proximidades de los sitios arqueológicos. Esto debe facilitar la realización de intervenciones e investigaciones arqueológicas. Se debe sumar la deficiencia de carreteras y caminos en toda el área. Por último, la séptima prioridad en orden de calificación fue la intervención para la conservación de las colecciones arqueológicas existentes (30 puntos). Si bien la valoración general es baja, esto se debe a que la mayoría de los sitios no cuentan con colecciones arqueológicas, sin embargo, aquéllas existentes en Kuélap y en La Jalca Grande requerían de intervención urgente.

\section{CALIficación final}

\subsection{Selección y prioridades de intervención por potencial de los sitos.}

Para el establecimiento de la calificación final de cada uno de los sitios y seleccionar la prioridad de intervención en términos del conjunto, se procedió de la siguiente manera: se sumaron los puntajes de indicador de potencial y de las prioridades de intervención en cada sitio. Luego se estableció el promedio entre ambos puntajes y la diferencia obtenida de la resta entre el promedio más alto y el más bajo (34) se dividió entre cuatro (8.5) a fin de separar los puntajes en el mismo número de niveles de prioridad (baja, media, alta y muy alta). El resultado de la división marca los puntos de separación entre cada uno de los niveles a partir del promedio inferior hasta el más alto.

Esta calificación, aunque es siempre referencial, deja ver el comportamiento general del conjunto de los sitios, pero además, fue útil para definir prioridades de intervención una vez que estos fueron organizados como parte de las unidades geohistóricas. En ese caso, puede darse que un sitio que en esta calificación ocupe un nivel bajo de prioridad, en el interior de su unidad Geohistórica se ubique en un nivel de prioridad más alto que los otros sitios que también la conforman. Es también importante notar, que puede haber aspectos puntuales de calificación con un puntaje muy alto de importancia pero que fueron relativizados por las bajas calificaciones en otros aspectos, haciendo que algunos sitios a pesar de su importancia se ubiquen en un nivel Bajo de calificación. Tómese también en cuenta que todos los sitios fueron calificados con una máxima necesidad de intervenciones de conservación (9 puntos), pero que éstas, finalmente, se supeditan también a los otros objetivos del PMK.

\section{2. Áreas de Paisajes Agroarqueológicos, naturales y sitios asociados}

Como criterio de acción del PMK se buscaba conservar la estructura paisajística del área e integrar los diversos atractivos por medio de los mismos caminos usados en la antigüedad. Si bien 


\begin{tabular}{|l|c|c|c|c|c|}
\hline \multicolumn{7}{|c|}{ Figura 15. Prioridad de intervención por sitio } \\
\hline Sitio & $\begin{array}{c}\text { Indicador de } \\
\text { Potencial }\end{array}$ & $\begin{array}{c}\text { Prioridades de } \\
\text { intervención }\end{array}$ & Total & Promedio & Calificación \\
\hline 1. Kuélap & 90 & 41 & 131 & 65.5 & Muly alto \\
\hline 2. Revash & 69 & 37 & 106 & 53 & \\
\hline 3. Casa Redonda & 65 & 39 & 104 & 52 & \\
\hline 4. Olán & 66 & 37 & 103 & 51.5 & Alto \\
\hline 5. Ollape & 61 & 37 & 98 & 49 & \\
\hline 6. La Congona & 56 & 27 & 83 & 41.5 & Medio \\
\hline 7. Tuich/Silic & 45 & 35 & 80 & 40 & \\
\hline 8. Ingapirca & 45 & 33 & 78 & 39 & \\
\hline 9. Tella & 50 & 27 & 77 & 38.5 & \\
\hline 10. Macro & 41 & 33 & 74 & 37 & \\
\hline 11. Yuructuna & 41 & 31 & 72 & 36 & \\
\hline 12. Machupirca & 38 & 29 & 67 & 33.5 & \\
\hline 13. Pumachaca & 32 & 33 & 65 & 32.5 & \\
\hline 14. Cataneo & 38 & 25 & 63 & 31.5 & \\
\hline 15. El Molinete & 38 & 25 & 63 & 31.5 & Bajo \\
\hline
\end{tabular}

todos los sitios se asociaban a hermosas vistas paisajísticas, era de primera importancia considerar los paisajes agroarqueológicos y los naturales (relictos), junto a los caminos prehispánicos y a los sitios arqueológicos asociados, pues en estos casos se elevaba el potencial de aprovechamiento. Se puede citar (figuras 16 y 17) las áreas de andenes de La Jalca, asociadas al camino Inca Ubilón - Zuta y al camino de herradura de La Jalca a Ubilón; las áreas de andenes en Lirio y San José en Kuélap como parte del paisaje inmediato de Kuélap y el poblado arqueológico de Imperio parte del uso de esta infraestructura; las áreas de andenes de Mariscal Castilla asociadas al sitio arqueológico de Olán; el sitio de Shucsho, que no es de gran potencial arqueológico pero sí paisajístico, por su vista de los relictos de bosques que dominan las montañas hasta Leymebamba pudiéndose también apreciar La Congona. Un área de andenes cercana en la Quebrada El Silencio debía ser posteriormente evaluada.

Para el caso, se usó la evaluación de indicadores de potencial pues la priorización de ac- tividades requerían más trabajo de campo. Las calificaciones de los sitios incluidos en este rubro son bastante homogéneas entre sí, con la excepción del sitio de Shucsho. Se propuso en el PMK que la intervención en estos sitios fuera intensa y sostenida durante los 10 años.

\section{LAS UnidAdes GeOHISTÓRICAS}

\subsection{Aspectos generales}

Como producto de la discusión del equipo multidisciplinario del PMK se definieron 8 unidades geohistóricas (UGH) y 6 unidades de reserva. Las unidades geohistóricas son subdivisiones territoriales al interior del área del PMK. En ellas confluyen los restos arqueológicos, hechos históricos, formaciones naturales y los elementos de la cultura viva tradicional dando un carácter singular y único a cada una. A pesar de esta singularidad, cada unidad geohistórica es parte de una unidad procesal mayor que permitía articular como una unidad al conjunto del área. En el caso arqueológico, el área del PMK es una 


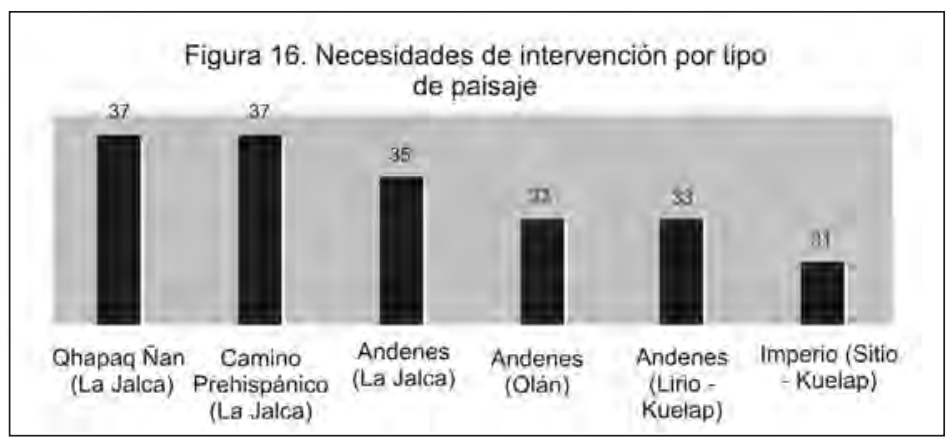

unidad multiétnica prehispánica en la cual los procesos de desarrollo social se dieron de forma integrada.

Los restos arqueológicos de cada UGH son de diversos tipos y se estructuraban como parte de la organización socio-productiva a nivel macro y micro territorial al interior del área del PMK. Los tipos de restos arqueológicos del área se organizaron de la siguiente manera: Aldeas principales (Kuelap, Olán); Aldeas de segunda jerarquía (Tella, Ollape); Aldeas de tercera jerarquía (La Congona, Cataneo, Molinete, Shucsho, Sec'sto, Putquero Loma, Tuich, Macro, Machupirca, Yuructuna); Paisajes agroarqueológicos (Kuélap, La Jalca, Mariscal Castilla, Montevideo, otros por definir); Bosques relicto arqueológico (en toda el área del PMK); Caminos incaicos y preincaicos (Ubillón Suta, La Jalca - Ubillón, Magdalena - Levanto); Puentes (Nogalcucho/Utcubamba, Levanto/Magdalena); Escultura en piedra (Pumachaca).

Por motivo de la excesiva extensión de la fundamentación y descripción de las UGH, la cual puede ser consultada en los informes presentados al MINCETUR y al INC de Lima, nos interesa para los fines del presente artículo, mencionar cual fue su tratamiento metodológico en términos de

\begin{tabular}{|c|c|}
\hline \multicolumn{2}{|l|}{ Figura 18. UGH La Jalca } \\
\hline Sitios & Puntaje \\
\hline Casa redonda & 39 \\
\hline Ollape & 37 \\
\hline Museo Arqueológico Etnográfico -Histórico & 35 \\
\hline Camino La Jalca - Ubillón & 35 \\
\hline Paisaje Agroarqueológico Singache - Saucuhuayco - La Jalca & 35 \\
\hline Cuyunahuayco & 35 \\
\hline Sec'sto & 0 \\
\hline Putquero Loma & 0 \\
\hline Promedio & 36 \\
\hline
\end{tabular}

Figura 16. . Necesidades de intervención por tipo de paisaje

la aplicación del sistema de valoración descrito hasta el momento. Para ello, presentamos acá los sitios arqueológicos que componen cada UGH y los puntajes de su valoración de prioridad de intervención. En cada UGH se estableció un promedio de la suma de todos los puntajes obteniéndose un nuevo valor que nos permitía establecer cuál es la relación de importancia entre cada UGH y cuales eran más importante intervenir en base a los objetivos del PMK.

Las unidades geohistóricas identificadas se califican en las figuras del 17 al 23:

\begin{tabular}{|l|c|}
\hline \multicolumn{2}{|c|}{ Figura 17. UGH Kuélap } \\
\hline Sitios & Puntaje \\
\hline Kuélap & 41 \\
\hline San José & 33 \\
\hline El Lirio & 33 \\
\hline Imperio & 31 \\
\hline \multicolumn{1}{|c|}{ Promedio } & 34.5 \\
\hline
\end{tabular}

Se definió también la Unidad Geohistórica Leymebamba cuyo eje principal era al centro poblado de Leymebamba y el Museo Mallqui, pero la temática expositiva eran principalmente los materiales arqueológicos provenientes de los mausoleos de la Laguna de los Cóndores. No se registraron sitios arqueológicos para la Unidad, sin embargo, se debía esperar hasta la realización de mayores 


\begin{tabular}{|c|c|}
\hline \multicolumn{2}{|l|}{ Figura 19. UGH Olán } \\
\hline Sitios & Puntaje \\
\hline Olán & 37 \\
\hline $\begin{array}{l}\text { Area de Paisaje Agroarqueológico: } \\
\text { Quebrada Cunche- Quebrada Co- } \\
\text { rralhuayco - Cerro Olán }\end{array}$ & 33 \\
\hline Shucsho & 21 \\
\hline Alcalde Urco & 16 \\
\hline Monja & 0 \\
\hline Carpona & 0 \\
\hline Palpata & 0 \\
\hline Arica & 0 \\
\hline Monja & 0 \\
\hline Promedio & 26.75 \\
\hline
\end{tabular}

\begin{tabular}{|l|c|}
\hline \multicolumn{2}{|c|}{ Figua 20. UGH Revash } \\
\hline Sitios & Puntaje \\
\hline Revash (mausoleo principal) & 37 \\
\hline $\begin{array}{l}\text { Sitios solo Identificados } \\
\text { (Mausoleos en Carangote, } \\
\text { Cerro Carbón, otros) }\end{array}$ & 0 \\
\hline Promedio & 37 \\
\hline
\end{tabular}

\begin{tabular}{|l|c|}
\hline \multicolumn{2}{|c|}{ Figura 21. UGH Ribera del Utcubamba } \\
\hline Sitios & Puntaje \\
\hline CapacNan: Ubillón - Zuta & 35 \\
\hline Capac Ñan Magdalena - Levanto & 35 \\
\hline Silic/Tuich & 35 \\
\hline Inkapirka & 33 \\
\hline Macro & 33 \\
\hline Pumachaca & 33 \\
\hline Yuructuna & 31 \\
\hline Machupirca & 29 \\
\hline \multicolumn{2}{c}{ Promedio } \\
\hline
\end{tabular}

exploraciones. Se caracteriza por ser un punto de transición entre Cajamarca y el Utcubamba, característica que pudo tener su antecedente en antiguos límites étnicos en esta parte de la cuenca. El Museo Mallqui era el único recurso arqueológico registrado para Leymebamba al
Figura 22. UGH San Francisco del Yeso - La Congona

\begin{tabular}{|c|c|}
\hline Sitios & Puntaje \\
\hline La Congona & 27 \\
\hline Cataneo* & 25 \\
\hline Molinete* & 25 \\
\hline Challapunta* & 0 \\
\hline Promedio & 25.66 \\
\hline \multicolumn{2}{|l|}{ * Promedio preliminar } \\
\hline
\end{tabular}

\begin{tabular}{|l|c|}
\hline \multicolumn{2}{|c|}{ Figura 23. UGH Tella - Cavernas } \\
\hline Sitios & Puntaje \\
\hline Tella & 27 \\
\hline Caverna Shihual & 19 \\
\hline Cosharán & 0 \\
\hline Plaza Pampa & 0 \\
\hline Cavernas Gupjie & 0 \\
\hline \multicolumn{2}{|r|}{ Promedio } \\
\hline
\end{tabular}

momento de la realización del PMK. Por esta razón no se incluyó en las UGH.

\subsection{Análisis de los cuadros. Elementos para la priorización de las unidades.}

Los promedios se establecen sólo para los sitios que cuentan con información previa con base al número de sitios calificados. Las unidades de mayor cantidad de sitios arqueológicos (La Jalca, Revash, Olán y Kuelap) son las que tienen también mayor promedio de potencial de intervención.

A continuación se propuso la organización de las unidades según su orden de importancia a partir de la puntuación y de criterios cualitativos en base a las observaciones de campo y de las fuentes. Las unidades se organizan en orden de importancia de la siguiente manera:

La Unidad Kuelap no contaba con un gran número de sitios arqueológicos (6 hasta el momento) cuyos potenciales fueran altos por sí mismos, sino por la ubicación en el área de influencia de este gran sitio arqueológico. Por lo 
tanto, y dado que Kuélap es el sitio eje del PMK, su intervención es urgente y prioritaria más allá de las cifras. Su calificación de importancia en comparación con el resto de las unidades es primera. En el mismo nivel de importancia se sitúa la necesidad de restituir la infraestructura que conformaba su paisaje agrícola y la forma en que este se explotó. Las obras de habilitación de servicios y mejoramiento de senderos y circuitos de visita se calificaron de urgente. Se debía intervenir en el corto plazo del PMK.

La Unidad Geohistórica de las riberas del Utcubamba es de una mayor importancia a la que revela su promedio. Aparte de constituir una unidad medio ambiental (riberas y río) bien definida, al igual que arqueológicamente (Ocupación inca, sitios Chachapoyas para el control del valle inferior) cumplía y también actualmente la función de articular a los pueblos del área mediante la principal carretera que une Chachapoyas con Leymebamba y Cajamarca. La Unidad se constituye en el soporte del acceso, introducción y flujo al resto de Unidades Geohistóricas. Por lo tanto, la intervención en los sitios arqueológicos que la conforman pueden tener un impacto inmediato sobre el conjunto del área (comprende a todos los distritos del PMK), teniendo en cuenta que la facilidad de su acceso debía "arrastrar" la visita a otros sitios del área. Se planteó que esta área fuera desarrollada por el PMK en forma más intensa desde el inicio del PMK. El conjunto de los sitios deben ser señalizados y sujetos a trabajos de conservación en el corto plazo del PMK.

La Unidad de La Jalca es la más homogénea. Es decir tiene un gran número de sitios arqueológicos y al mismo tiempo un promedio muy alto y muy bien distribuido entre el conjunto de sitios arqueológicos que la conforman e incluye inclusive la presencia de colecciones arqueológicas relevantes. Su calificación en importancia debería ser en segundo lugar. Los sitios de mayor interés a intervenir en el corto plazo fueron Casa Redonda, y Ollape. Mientras que las áreas agroarqueológicas y el camino preincaico Ubilón - Zuta debían ser tratados como una unidad en el mediano y largo plazo. El resto de sitios convenía ser incluidos como parte de las tareas de exploración en el largo plazo. El trabajo con las colecciones arqueológicas debía darse, más bien, desde el inicio del PMK en forma sostenida.

Olán es un sitio arqueológico muy importante, tanto como Kuelap, sin embargo, su alta valoración se diluye en el promedio de las otras $\mathrm{UGH}$, ante la presencia de otros sitos en la zona cercana con baja calificación (Shucsho, Alcalde Urco). Sin embargo, en las evaluaciones de potencial de intervención y de tipo de actividades este sitio, siempre se alcanzó un puntaje muy alto. Es más, si consideramos el promedio sólo de los dos sitios más importantes (Olán $\{37\}$ y las áreas de andenes $\{33\}$ ) nos da un promedio de 35 dejando ver en forma más real el verdadero y gran potencial de esta Unidad Geohistórica. Se puede considerar en tercera ubicación de importancia. Su intervención debía comprender Olán y el área agroarqueológica de Mariscal Castilla y darse en el corto plazo por ser de gran importancia. La intervención en el resto de sitios del área puede darse en el mediano largo plazo del PMK.

La Unidad de Revash es de la misma importancia que la de Olán. Se supone que en la cuenca del río ingenio hay más de 50 mausoleos (cerro Carbón y Carangote) lo que le da a la unidad una extensión considerable tomando en cuenta la distribución de estos en una gran área natural de formaciones geológicas y vegetales de gran belleza. La singularidad de las estructuras funerarias le da a la Unidad un valor especial pues permitía contar con una "oferta" distinta al patrón arquitectónico en serie del resto de las aldeas arqueológicas (aldeas con casas circulares de piedra, organizadas sobre terrazas constructivas en las partes altas y medias de los cerros). Junto a esta importancia, el mal estado de conservación, el saqueo y vandalismo así como el riesgo mortal de la realización de visitas no controladas a los mausoleos, motivaba que fuera urgente incorporar la intervención a las actividades del PMK para el corto y mediano plazo. 
La Unidad Geohistórica de San Francisco del Yeso se conformó por asentamientos arqueológicos de menor jerarquía (esto no quiere decir que estos no sean importantes en su significado y arquitectura) o a que no se contaba con información suficiente de los sitios que las componen. Se propuso la intervención en La Congona, como sitio arqueológico complementario a la Unidad Geohistórica de Leymebamba (a pesar de estar en otro distrito: San Francisco del Yeso), en el corto plazo del proyecto. El resto de sitios debe ser parte de los proyectos exploratorios más intensos propuestos.

La Unidad Geohistórica Tella - Cavernas de Magdalena, al igual que en el caso anterior, demandaba de una mayor exploración. Sin embargo, En el caso de Tella, se producía el mismo fenómeno de Olán. Es un sitio de gran importancia arqueológica pero la baja calificación de Shihual hacía que su promedio bajara. No obstante, su importancia no podía ser dejada de lado, pues en Tella se puede realizar la conexión visual con toda el área que se extiende al norte del PMK (San Isidro del Mayno). Se propuso que la intervención en el sitio se diera en el mediano y largo plazo ya que otros sitios ubicados en el distrito de Magdalena y cerca de Tella se intervendrían desde el comienzo como parte de la Unidad Geohistórica Riberas del Utcubamba.

\begin{tabular}{|l|c|}
\hline \multicolumn{2}{|c|}{$\begin{array}{c}\text { Figura 24. Promedios de potencial de interven- } \\
\text { ción por UGH }\end{array}$} \\
\hline Sitios & Promedios \\
\hline Revash & 37 \\
\hline La Jalca & 36 \\
\hline Kuelap & 35 \\
\hline Riberas del Utcubamba & 33 \\
\hline Olán & 27 \\
\hline San Francisco del Yeso & 26 \\
\hline Cavernas Magdalena & 23 \\
\hline
\end{tabular}

\begin{tabular}{|c|c|}
\hline \multicolumn{2}{|c|}{$\begin{array}{c}\text { Figura } 25 . \mathrm{N}^{\circ} \text { de sitios arqueológicos por Uni } \\
\text { dad Geo-Histórica }\end{array}$} \\
\hline UGH & № Sitios \\
\hline La Jalca & 8 \\
\hline Riberas del Utcubamba & 8 \\
\hline Olán & 8 \\
\hline Kuelap & 6 \\
\hline San Francisco del Yeso & 4 \\
\hline Cavernas Magdalena & 2 \\
\hline Revash & 1 \\
\hline Total & 37 \\
\hline
\end{tabular}

\section{INTERVENCIONES Y CRONOGRAMA}

Una vez obtenidos estos valores, complementados y reordenados a partir de las observaciones de campo y de los aspectos no medidos o no medibles, la meta consistía en programar las intervenciones en cada sitio en forma de módulos que permitiesen establecer tipos de intervención por sitio, por UGH y por año (Figura 26) así como el número de intervenciones a lo largo de todo el tiempo de duración del PMK (Figura 27). Esto era importante pues cada módulo o Tipo de intervención podía ser presupuestado sobre costos fijos facilitándose mucho el cálculo de las inversiones y de los presupuestos, tarea complicada especialmente cuando se trata de un número alto de sitios arqueológicos y plazos largos. Es justamente en base a la tabla final de la figura 27 sobre la que se podía finalmente elaborar el costo total del plan.En base a estos criterios se definieron tres tipos de intervenciones distintas para los sitios arqueológicos y una para las colecciones arqueológicas:

Intervención de Tipo I: exploración (identificación); limpieza; delimitación / negociación propietarios; expediente técnico (elaboración, evaluación); intervenciones urgentes de conservación y excavación; recolección de materiales superficiales. 


\begin{tabular}{|c|c|c|c|c|c|c|c|c|c|c|c|c|}
\hline \multicolumn{13}{|c|}{ Figura 26. Tipos de intervención por UGH, por sitio y por año } \\
\hline & & \multicolumn{11}{|c|}{ Años* } \\
\hline Unidad & Sitios & 1 & 2 & 3 & 4 & 5 & 6 & 7 & 8 & 9 & 10 & \\
\hline \multirow[t]{8}{*}{ Kuélap } & Kuélap & III & & & & & & & & & & \\
\hline & San José & I & II & & & & & & & & & \\
\hline & Lirio & I & II & & & & & & & & & \\
\hline & Imperio & & & I & II & & & & & & & \\
\hline & América & & & I & II & & & & & & & \\
\hline & Santa Clara & & & I & & II & & & & & & \\
\hline & Sitios sin registro & & & & & & & & & & & \\
\hline & Museo /centro de Inv. & & & & & & & & & & & \\
\hline \multirow[t]{10}{*}{ R.Utcubamba } & Macro & I & II & & & & & & III & & & \\
\hline & Yuructuna & & I & & & & II & & & & & \\
\hline & Machupirca & & I & & & & II & & & & & \\
\hline & Tuich/Silic & I & II & & & & & & & & & \\
\hline & Pumachaca & I & II & & & & & & & & & \\
\hline & Qapac Nan - Mag. & & I & II & & & & & & & & \\
\hline & Qapac Ñan - L.Jalca & I & II & & III & & & & & & & \\
\hline & Ingapirka & & I & II & & & & & & & & \\
\hline & Sitios sin registrar & & & & & & & & & & & RESERVA \\
\hline & Centro. Inf. Municipal & & & & & & & & & & & \\
\hline \multirow[t]{8}{*}{ La Jalca } & Casa Redonda & I & II & & & & & & & & & \\
\hline & Ollape & & I & II & & & III & & & & & \\
\hline & Andenes (2) & I & II & & & & & & & & & \\
\hline & Camino Preinca & & I & II & & & & & & & & \\
\hline & Sec'sto & & & I & & & & & & & & RESERVA \\
\hline & Putquero Loma & & & I & & & & & & & & RESERVA \\
\hline & Sitios sin registrar & & & & & & & & & & & RESERVA \\
\hline & Museo Arq. antr. hist. & & & & & & & & & & & \\
\hline
\end{tabular}

Intervención de Tipo II: limpieza / mantenimiento temporal /ornato y embellecimiento; excavaciones exploratorias (cronología, prospección arquitectónica, recolección de materiales); conservación temporal; circuitos de visita; señalización.
Intervención de tipo III: limpieza y mantenimiento permanente; excavaciones intensivas; conservación permanente / intervenciones urgentes / exposición de estructuras; Análisis y conservación de materiales arqueológicos; habilitación 


\begin{tabular}{|c|c|c|c|c|c|c|c|c|c|c|c|c|}
\hline \multicolumn{13}{|c|}{ Figura 26. Tipos de intervención por UGH, por sitio y por año } \\
\hline & & \multicolumn{11}{|c|}{\begin{tabular}{|c|} 
Años* \\
\end{tabular}} \\
\hline Unidad & Sitios & 1 & 2 & 3 & 4 & 5 & 6 & 7 & 8 & 9 & 10 & \\
\hline \multirow[t]{8}{*}{ Kuélap } & Kuélap & III & & & & & & & & & & \\
\hline & San José & I & II & & & & & & & & & \\
\hline & Lirio & $\mathrm{I}$ & II & & & & & & & & & \\
\hline & Imperio & & & $\mathrm{I}$ & II & & & & & & & \\
\hline & América & & & I & II & & & & & & & \\
\hline & Santa Clara & & & $\mathrm{I}$ & & II & & & & & & \\
\hline & Sitios sin registro & & & & & & & & & & & \\
\hline & Museo /centro de Inv. & & & & & & & & & & & \\
\hline \multirow[t]{10}{*}{ R.Utcubamba } & Macro & $\mathrm{I}$ & II & & & & & & III & & & \\
\hline & Yuructuna & & I & & & & II & & & & & \\
\hline & Machupirca & & I & & & & II & & & & & \\
\hline & Tuich/Silic & $\mathrm{I}$ & II & & & & & & & & & \\
\hline & Pumachaca & I & II & & & & & & & & & \\
\hline & Qapac Nan - Mag. & & I & II & & & & & & & & \\
\hline & Qapac Ñan - L.Jalca & I & II & & III & & & & & & & \\
\hline & Ingapirka & & I & II & & & & & & & & \\
\hline & Sitios sin registrar & & & & & & & & & & & RESERVA \\
\hline & Centro. Inf. Municipal & & & & & & & & & & & \\
\hline \multirow[t]{8}{*}{ La Jalca } & Casa Redonda & $\mathrm{I}$ & II & & & & & & & & & \\
\hline & Ollape & & $\mathrm{I}$ & II & & & III & & & & & \\
\hline & Andenes (2) & $\mathrm{I}$ & II & & & & & & & & & \\
\hline & Camino Preinca & & $\mathrm{I}$ & II & & & & & & & & \\
\hline & Sec'sto & & & $\mathrm{I}$ & & & & & & & & RESERVA \\
\hline & Putquero Loma & & & $\mathrm{I}$ & & & & & & & & RESERVA \\
\hline & Sitios sin registrar & & & & & & & & & & & RESERVA \\
\hline & Museo Arq. antr. hist. & & & & & & & & & & & \\
\hline \multirow[t]{2}{*}{ Revash } & Mausoleo Principal & I & II & III & & & & & & & & \\
\hline & Sitios por registrar & & & II & & & & & & & & \\
\hline \multirow[t]{5}{*}{ Olán } & Olán & $\mathrm{I}$ & II & & III & & & & & & & \\
\hline & Andenes (2) & & I & II & & & & & III & & & \\
\hline & Arica & & & $\mathrm{I}$ & & & II & & & & & \\
\hline & Monja & $\mathrm{I}$ & & & & II & & & & & & \\
\hline & Shucsho & & & $\mathrm{I}$ & & & & & II & & & \\
\hline
\end{tabular}




\begin{tabular}{|l|l|l|l|l|l|l|l|l|l|l|l|l|}
\hline & \multicolumn{7}{|c|}{ Años } \\
\hline & Carpona & & & I & & & & & & & & RESERVA \\
\hline & Sitios sin registro & & & & & & & & & & & RESERVA \\
\hline & Centros Inf. Municipal & & & & & & & & & & & $(2)$ \\
\hline S.F. del Yeso & La Congona & I & II & & & & & & III & & & \\
\hline & Cataneo & & I & & & II & & & & & & \\
\hline & Molinete & & & I & & II & & & & & & \\
\hline & Challuapunta & & I & & & & & & & & & RESERVA \\
\hline & Sitios sin registrar & & & & & & & & & & & RESERVA \\
\hline Tella & Tella & I & & & & & & & & & & RESERVA \\
\hline & Shihual & & I & & & & & & & & & RESERVA \\
\hline & Sitios sin registrar & & & & & & & & & & & RESERVA \\
\hline * Las intervenciones tipo se mantienen a lo largo de los 10 años
\end{tabular}

de áreas y servicios: señalización, centro de información, parque biológico expositivo, circuitos de visita, áreas para depósito, investigación y conservación, áreas recreativas y educativas. Intervención de Tipo IV: identificación de colecciones, inventario y registro, conservación, análisis arqueológico, almacenaje, exposiciones permanentes y temporales, habilitación de infraestructura, recepción de nuevas colecciones

\section{COMENTARIOS Finales}

Se han presentado resumidamente los principales aspectos del sistema de valoración de monumentos arqueológicos que se propuso para el PMK en el año 2003. Debe tomarse en cuenta que no se encontraron referencias previas al uso de un método parecido para la elaboración de planes de manejo de sitios arqueológicos por lo que se trató de una primera exploración experimental de la posibilidad de usar métodos ordenados y sistematizados para este tipo de proyectos.

Por esta razón, el sistema presentaba debilidades, tal como puede notarse, que ocasionaron algunos desajustes metodológicos. Las categorías usadas no siempre expresaron bien las problemáticas y posiblemente, los puntajes obtenidos no siempre expresaron correctamente las realidades evaluadas. Con todo, cabe decir que hay aspectos no medibles o cuantificables que hacen imprescindible el uso de las observaciones de campo como complemento de las mediciones. Definitivamente, no es posible pensar en un método en el que la cuantificación sea el único parámetro de la evaluación y siempre las observaciones de campo del investigador deberán primar. Pero, y allí radica justamente la importancia del método, es que el investigador deberá sustentar en contra de una serie de variables que le indican lo contrario el porqué de sus decisiones. $\mathrm{O}$, por el contrario, deberá sustentar en base a sus conocimientos y experiencias de campo porqué sí, la cuantificación está expresando correctamente una realidad concreta.

Finalmente, a pesar del tiempo transcurrido desde la elaboración de los insumos arqueológicos que conformaron el PMK, el fin de este artículo ha sido presentar a la comunidad académica un sistema de valoración de monumentos que nos haga pensar en requerimientos más estandarizados en el registro, evaluación, planificación y gestión del patrimonio arqueológico los cuales se someten ahora a la crítica académica. 


\begin{tabular}{|c|c|c|c|c|c|c|c|c|c|c|c|c|}
\hline \multicolumn{13}{|c|}{ Figura 27. Cantidad de intervenciones tipo por año } \\
\hline Unidad & Intervenciones Tipo & 1 & 2 & 3 & 4 & 5 & 6 & 7 & 8 & 9 & 10 & \\
\hline \multirow[t]{4}{*}{ Kuélap } & I & 2 & & 3 & & & & & & & & 5 \\
\hline & II & & 2 & & 2 & 2 & & & & & & 6 \\
\hline & III & 1 & & & & & & & & & & 1 \\
\hline & Sub total & 3 & 2 & 3 & 2 & 2 & & & & & & 12 \\
\hline \multirow[t]{4}{*}{ R.Utcubamba } & I & 4 & 2 & 2 & & & & & & & & 8 \\
\hline & II & & 4 & 2 & & & & & & & & 6 \\
\hline & III & & & & 1 & & & & 1 & & & 2 \\
\hline & Sub total & 4 & 6 & 4 & 1 & & & & 1 & & & 16 \\
\hline \multirow[t]{4}{*}{ La Jalca } & $\mathrm{I}$ & 2 & 2 & 2 & & & & & & & & 6 \\
\hline & II & & 2 & 2 & & & & & & & & 4 \\
\hline & III & & & & & & 1 & 1 & & & & 2 \\
\hline & Sub total & 2 & 4 & 4 & & & 1 & 1 & & & & 12 \\
\hline \multirow[t]{4}{*}{ Revash } & $\mathrm{I}$ & 1 & & & & & & & & & & \\
\hline & II & & 1 & & 1 & & & & & & & \\
\hline & III & & & & 1 & & & & & & & 1 \\
\hline & Sub total & 1 & 1 & & 2 & & & & & & & 4 \\
\hline \multirow[t]{4}{*}{ Olán } & I & 1 & 1 & 3 & & & & & & & & 5 \\
\hline & II & & 1 & 1 & & 1 & 1 & & 1 & & & 5 \\
\hline & III & & & & & 1 & & & 1 & & & 2 \\
\hline & Sub total & 1 & 2 & 4 & & 2 & 1 & & 1 & & & 13 \\
\hline \multirow[t]{4}{*}{ S.F. del Yeso } & I & 1 & 1 & 2 & & & & & & & & 4 \\
\hline & II & & 1 & & & 2 & & & & & & 3 \\
\hline & III & & & & & & & & 1 & & & 1 \\
\hline & Sub total & 1 & 2 & 2 & & 2 & & & 1 & & & 8 \\
\hline \multirow[t]{4}{*}{ Tella-Cavs. } & I & 1 & 1 & & & & & & & & & 2 \\
\hline & II & & & & & & & & & & & \\
\hline & III & & & & & & & & & & & \\
\hline & Sub total & 1 & 1 & & & & & & & & & 2 \\
\hline TOTAL & & 13 & 18 & 17 & 5 & 6 & 2 & 1 & 2 & 0 & 0 & 68 \\
\hline
\end{tabular}

\section{BibLIOGRAFÍA}

Ángeles, Andrés, Jorge Araujo, Carlos Melendez, Niryam Meza y Ludecinio Alvarado

1998 Registro arqueológico de la Provincia de Chachapoyas, Distrito Montevideo. Informe del trabajo para optar el título de Profesor. Instituto Tecnológico Superior Pedagógico "Toribio Rodriguez de Mendoza". Sub Región V - Educación. Chachapoyas Amazonas Perú.

Bardales, Manuel

2002 Breve reseña histórica y recursos del distrito en turismo. Distrito de María. 
Cabañas, Jacobita, Jhulisa Collantes, Gilma Hidalgo, Raquel Velásquez y Pedro Reina

1998 Registro arqueológico de la Provincia de Chachapoyas, Distrito Mariscal Castilla. Informe del trabajo para optar el título de Profesor. Instituto Tecnológico Superior Pedagógico "Toribio Rodríguez de Mendoza”. Sub Región V - Educación. Chachapoyas. Amazonas Perú.

Camino, Alejandro y Carlos Dávila

1981 "Bibliografía de la Arqueología de la Amazonía Peruana”. Amazonía Peruana. Vol III, n8.

Chirinos, Ricardo

s/f Informe Pericial de la Zona Arqueológica de Machupirca - distrito de Magdalena. Instituto Nacional de Cultura. Impreso.

COMINCO

2001 Expediente de Laguna de los Cóndores y CIRA (Certificado de Inexistencia de Restos Arqueológicos).

Culqui, José

1999 Estudio del Capac Nan en la cultura Chachapoya. Informe del trabajo de investigación para optar el Título de Profesor, especialidad: Historia y Geografía. Instituto Superior Pedagógico "Toribio Rodríguez de Mendoza" CTAR A-D-R- Educación. Chachapoyas.

Corbero, Herman

1987 "Estudio del suelo y delimitación del Área de Protección y Reserva del complejo arqueológico de Kuelap". Ms. Chachapoyas. INC - Amazonas.

Davis, Morgan

1985 Chachapoyas. The Cloud People. An anthropological survey (Ed.mim.) Ontario.

1996 La Casa Redonda and Yurac-Urco: two round houses in department Amazonas, Perú. Noelville, Ontario.
Feilden, Bernard y Jukka Jokilehto

1995 Manual para el manejo de los Sitios Culturales del Patrimonio Mundial. Instituto Colombiano de Cultura - Colcultura. Dirección de Patrimonio. Bogotá.

García, Dalila, María Goñas, Rodolfo Huamán, Demetrio Sánchez y Nery López

1998 Registro arqueológico de la Provincia de Chachapoyas, Distrito Jalca Grade. Informe del trabajo para optar el título de Profesor. Instituto Tecnológico Superior Pedagógico "Toribio Rodríguez de Mendoza”. Sub Región V - Educación. Chachapoyas Amazonas. Perú.

Gil, Napoleón

1938 "Dos pueblos prehistóricos kuelapenses: Kacta y Chipuric". Boletín de la Sociedad Geográfica de Lima 55: 2-3, 132-139.

Guillén, Sonia

(1998) Arqueología de emergencia: inventario, catalogación y conservación de los materiales arqueológicos de los mausoleos de la Laguna de los Cóndores. Informe entregado al INC. Lima.

(1999) Evaluación y delimitación del sitio arqueológico Llaqtacocha. Informe entregado al INC. Lima.

Horkheimer, Hans

1959 "Algunas consideraciones de la arqueología del valle del Utcubamba". Actas y Trabajos del II Congreso Nacional de Historia del Perú 2: 71-95.

INC

2001 "Concesión del Título de Fidelísima Ciudad de Chachapoyas". Boletín N¹17 2000 INC, Amazonas.

2001 "Síntesis del Desarrollo histórico cultural de Chachapoyas (Batalla de Higos Urcos en 1821)”. Boletín $\mathrm{N}^{\circ} 117$ 2000 INC. Amazonas

Kauffmann, Federico

1980 "Los Pinchudos. Exploración de ruinas intactas en la selva". Boletin de 
Lima, n7. jul. Lima, pp26-31.

1984 "Pucullos y figuras antropomorfas de madera en el Antisuyo". Cielo Abierto 10: 29 : 45-52. Lima.

1986 "Sarcófagos pre-Incas en los Andes Amazónicos peruanos". Kuntur 1: 4 9. Lima.

1991 "Sobrepoblación en los Andes: una explicación del origen y proceso de la cultura andina”. L'Imaginaire 1 (3): 45-48. Publicación trimestral de la Alianza Francesa. Lima.

Kauffmann, Federico y Giancarlo Ligabue

1990 "Les Andes Amazoniennes". En : INCA-PERU 3000 ans d'Histoire : 262 - 275. Sergio Purin editor. Belgica.

1984 "Pucullos y figuras antropomorfas de madera en el Antisuyo". Cielo Abierto 10: 29:45-52. Lima.

Kauffmann, Federico, Miriam Salazar, Daniel Morales, Iain Mackay y Oscar Sacay

1989 "Andes Amazónicos: sitios intervenidos por la expedición Antisuyo/86". Arqueológicas 20. Lima: Instituto de Investigaciones Arqueológicas. Museo Nacional de Antropología y Arqueología.

Kieffer, Philippe

1910 Excursión a Cuelap (Departamento de Amazonas, Perú). E. Rosay, ed. Lima. Librería Francesa Científica

Langlois, Louis

1934 "Ruinas de Cuelap". Boletín de la Sociedad Geográfica. T.II, vol.1. Lima. pp: 20-34

1939 "Utcubamba". Revista del Museo Nacional del Perú tomo VIII, n², Lima. pp:224-240

1940 "Utcubamba”. (Continuación). Revista del Museo Nacional Tomo IX $\mathrm{n}^{\circ} 1$, 1er semestre, Lima. pp.: 33-72.

1040 "Utcubamba Investigaciones arqueológicos en este valle del departamento de Amazonas (Perú)”. (Conclusiones).
Revista del Museo Nacional, T.IX n², 2do. Semestre. Lima. pp. 191-228.

Morales Gamarra, Ricardo

2002 "Los Pinchudos. Arquitectura funeraria en río Abiseo, San Martín (Parte 1)". Arkinka 76: 92-101.

Morales Gamarra. Ricardo, L. Valle Alvarez, W. Church y L. Coronado Tello

2002 "Los Pinchudos: mausoleo polícromo de los Andes nor-orientales del Perú". Sian 8/12: 3-41. Trujillo.

Morgan, Davis

2001 "Diario de Viaje (Chachapoyas - Colcamar-Gran Vilaya)". En: Kuelap, Boletín Cultural, $\mathrm{N}^{\circ} 116$, INC Amazonas.

Mucipalidad Distrital de Colcamar

1999 "Evaluación turística del distrito". Expediente Técnico.

Narvaez, Alfredo

1986 "Rasgos arquitectónicos de la Fortaleza de Kuelap. Kuelap". Boletín Cultural 66, Chachapoyas: INC - Amazonas.

1988 "Kuelap. Una ciudad fortificada en los Andes nororientales de Amazonas, Perú”. En: Arquitectura y Arqueología, V. Rangel ed. :15-142. Concytec. Chiclayo.

1996a "La fortaleza de Kuelap 1". Arkinka 12: 92-108.

1996b "La forteleza de Kuelap 2". Arkinka 13: 90-98

Narvaez, Alfredo y Ricardo Morales

1999 Informe técnico Proyecto Piloto Kuelap I. Chachapoyas. INC/Palacio de Gobierno.

Paz Sotero, Rocío

2001 Implicancias Arquitectónicas de un sitio Prehispánico en el valle de Utcubamba - Chachapoyas. Proyecto de investigación para optar el título de Licenciado en Arqueología. Universidad Nacional de Trujillo. Impreso. 
INRENA

1988 Plan Maestro del Santuario Histórico de Machu-Picchu. Lima.

\section{UNESCO-INC}

2000 Planes de manejo de las Lineas de Nasca. Elaborado por encargo del convenio UNESCO - INC sobre medidas de Emergencia para la Conservación de las Líneas y Geoglifos de Nasca. Luis G. Lumbreras Coordinador. Lima.

Ravines, Roger

1972 "Los caciques de Pausamarca: algo más sobre las étnias de Chachapoyas". En: Historia y Cultura 6: 217-247.

1982 "Yacimientos arqueológicos de la región nororiental del Perú". Amazonia Peruana 4: 7: 139-175.

Reichlen, Henri et Paule

1950 "Recherches archéologiques dans les andes du haut Utcubamba". Journal de la Société del Américanistes, nouvelle série 34 : 219-250.

Rodriguez Eustaquio, Marco A.

2003 Informe de supervisión a los sitios arqueológicos del distrito de Colcamar. Informe $\mathrm{N}^{\circ}$ 008-2002/MARE/ARMAK/INC-A del 28/01/03.
Ruiz Estrada, Arturo

1985 "Los monumentos arqueológicos de Leimebamba". En: Boletín de Lima 7/42: 9-82.

Tafur Meza, Liborio

s/f Cuentos y leyendas de mi tierra. Impreso.

Torres Mas, Carlos y Alfredo Narvaez.

2000 "Proyecto de Investigación interdisciplinaria. INC Amazonas CORDE Amazonas". Kuelap, Boletín Cultural, № 113 .

Zubiate Zabarburu, Víctor

1984 Guía arqueológica del departamento de Amazonas. Chachapoyas, s/Ed.

1984 Zona Arqueológica del Departamento de Amazonas. Impreso.

2001 Recursos Turísticos del Departamento de Amazonas. Cámara de Promoción Turística, Leymebamba. Impreso. 